\title{
Subbiliazine: A contracted phthalocyanine analog
}

\author{
Briana R. Schrage, ${ }^{a}$ Victor N. Nemykin, ${ }^{\mathrm{b}}$ and Christopher J. Ziegler ${ }^{\mathrm{a}}$ \\ a Department of Chemistry, University of Akron, Akron, Ohio 44312-3601, United States \\ b Department of Chemistry, University of Tennessee, Knoxville, TN 37996, United States
}

\section{Supplementary Information}




\section{Table of Contents}

General Information

Full citation for Gaussian

Syntheses

Figure S1: ${ }^{1} \mathrm{H}$ NMR (300 MHz) of 1 in d6-DMSO.

Figure S2: ${ }^{1} \mathrm{H}$ NMR (300 MHz) of 2 in d6-DMSO.

Figure S3: ${ }^{1} \mathrm{H}$ NMR $(300 \mathrm{MHz})$ of $\mathbf{1 B F}$ in $\mathrm{CDCl}_{3}$.

Figure S4: ${ }^{1} \mathrm{H}$ NMR $(300 \mathrm{MHz})$ of $\mathbf{2 B F}$ in $\mathrm{CDCl}_{3}$.

Figure S5: ${ }^{1} \mathrm{H}$ NMR (300 MHz) of 3 in d6-DMSO.

Figure S6: ${ }^{13} \mathrm{C}\left\{{ }^{1} \mathrm{H}\right\}$ NMR $(125 \mathrm{MHz})$ of 1 in d6-DMSO.

Figure S7: ${ }^{13} \mathrm{C}\left\{{ }^{1} \mathrm{H}\right\}$ NMR $(125 \mathrm{MHz})$ of 2 in d6-DMSO.

Figure S8: ${ }^{13} \mathrm{C}\left\{{ }^{1} \mathrm{H}\right\} \mathrm{NMR}(125 \mathrm{MHz})$ of $\mathbf{1 B F}$ in $\mathrm{CDCl}_{3}$.

Figure 59: ${ }^{13} \mathrm{C}\left\{{ }^{1} \mathrm{H}\right\}$ NMR $(125 \mathrm{MHz})$ of $\mathbf{2} \mathbf{B F}$ in $\mathrm{CDCl}_{3}$.

Figure S10: ${ }^{13} \mathrm{C}\left\{{ }^{1} \mathrm{H}\right\}$ NMR $(125 \mathrm{MHz})$ of 3 in d6-DMSO.

Figure S11: ${ }^{19} \mathrm{~F}$ NMR (282 MHz) of $\mathbf{1 B F}$ in $\mathrm{CDCl}_{3}$.

Figure S12: ${ }^{19} \mathrm{~F}$ NMR (282 MHz) of $\mathbf{2 B F}$ in $\mathrm{CDCl}_{3}$.

Figure S13: ${ }^{19} \mathrm{~F}$ NMR (282 MHz) of 3 in d6-DMSO.

Figure S14: High-resolution ESI mass spectra of 1.

Figure S15: High-resolution ESI mass spectra of 2.

Figure S16: High-resolution ESI mass spectra of 1BF.

Figure S17: High-resolution ESI mass spectra of 2BF.

Figure S18: High-resolution ESI mass spectra of $\mathbf{3}$.

Figure S19: UV-visible spectra for compounds 1 and 2 in DMF.

Figure S20: UV-visible spectra for compounds $\mathbf{1 B F}$ and $\mathbf{2 B F}$ in $\mathrm{CHCl}_{3}$.

Figure S21: UV-visible spectra for compound 3 in DMF.

Figure S22: Absorption and excitation spectra for compounds $\mathbf{1 B F}$ and $\mathbf{2 B F}$ in $\mathrm{CHCl}_{3}$.

Figure S23: Cyclic voltammograms of 1, 2, 1BF, and 2BF in DMF/0.1 $\mathrm{TBAPF}_{6}$.

Figure S24: Structure of compound 1, with 35\% probability ellipsoids.

Figure S25: Structure of compound 2, with 35\% probability ellipsoids. 
Figure S26: Structure of compound 1BF, with 35\% probability ellipsoids. S35

Figure S27: Structure of compound 3, with 35\% probability ellipsoids. $\quad$ S36

Figure S28: Structure of compound 2BF, with 35\% probability ellipsoids. $\quad$ S37

Figure S29：DFT-predicted frontier orbitals for compounds 1 and $2 . \quad$ S38

Figure S30: DFT-predicted frontier orbitals for compounds 1BF and 2BF. S39

Figure S31: Experimental and B3LYP TDDFT-predicted spectra for $\quad$ S40 compounds 1, 2, 1BF, and 2BF.

Figure S32: Experimental B3LYP TDDFT-predicted excitation and emission spectra for compounds $\mathbf{1 B F}$ and $\mathbf{2 B F}$.

Figure S33: Relative energies of the frontier orbitals for $\quad$ S42 compounds $1,2,1 \mathrm{BF}$, and $2 \mathrm{BF}$.

Table S1: X-ray crystal data and structure parameters for compounds 1, and 2. $\quad$ S43

Table S2: X-ray crystal data and structure parameters for compounds 1BF, and 3. $\quad$ S44

Table S3: Selected bond lengths for compounds 1BF and 3. S45

Table S4: B3LYP TDDFT-predicted energies and expansion coefficients for $\quad$ S46 compound $\mathbf{1}$ (only excited states with $\mathrm{f}>0.05$ and $\lambda>270 \mathrm{~nm}$ are listed).

Table S5: B3LYP TDDFT-predicted energies and expansion coefficients for $\quad$ S46 compound 2 (only excited states with $\mathrm{f}>0.05$ and $\lambda>270 \mathrm{~nm}$ are listed).

Table S6: B3LYP TDDFT-predicted energies and expansion coefficients for compound $\mathbf{1 B F}$ (only excited states with $\mathrm{f}>0.05$ and $\lambda>270 \mathrm{~nm}$ are listed).

Table S7: B3LYP TDDFT-predicted energies and expansion coefficients for compound $\mathbf{2 B F}$ (only excited states with $\mathrm{f}>0.05$ and $\lambda>270 \mathrm{~nm}$ are listed).

Table S8: The first three B3LYP TDDFT-predicted emission energies and expansion coefficients for compound $\mathbf{1 B F}$

Table S9: The first three B3LYP TDDFT-predicted emission energies and expansion coefficients for compound $\mathbf{2 B F}$

Table S10: B3LYP DFT ground state optimized geometry of compound 1.

Table S11: B3LYP DFT ground state optimized geometry of compound 2.

Table S12: B3LYP DFT ground state optimized geometry of compound 1BF.

Table S13: B3LYP DFT ground state optimized geometry of compound 2BF.

Table S14: B3LYP DFT excited state optimized geometry of compound 1BF.

Table S15: B3LYP DFT excited state optimized geometry of compound 2BF. 


\section{General Information}

\section{Experimental}

All reagents and starting materials were purchased from commercial vendors and used without further purification. Deuterated solvents were purchased from Cambridge Isotope Laboratories and used as received.

NMR spectra were recorded on $300 \mathrm{MHz}$ and $500 \mathrm{MHz}$ spectrometers and chemical shifts were given in ppm relative to residual solvent resonances $\left({ }^{1} \mathrm{H}\right.$ NMR and ${ }^{13} \mathrm{C}$ NMR spectra). ${ }^{19} \mathrm{~F}$ NMR spectra were referenced and corrected to an external reference trifluoro acetic acid (TFA) standard (-76.55 ppm). High-resolution mass spectrometry experiments were performed on a Bruker MicroTOF-III and MicroTOF-qIII instruments. Infrared spectra were collected on Thermo Scientific Nicolet iS5 that was equipped with an iD5 ATR. UV-visible spectra were recorded on a Shimadzu UV-2600 UV-Visible spectrophotometer. Fluorescence emission data in solution were recorded on a Horiba Jobin-Yvon FluoroMax-4 fluorescence spectrophotometer using fluorescein in $0.1 \mathrm{M} \mathrm{NaOH}$ as a standard. All slit widths were held constant at $2 \mathrm{~nm}$. The quantum yields in solution were calculated using the following equation: $\Phi_{X}=\Phi_{S T}\left(\frac{\operatorname{Grad}_{X}}{\operatorname{Grad}_{S T}}\right) *\left(\frac{\eta_{X}^{2}}{\eta_{S T}^{2}}\right) ; \eta_{S T}=$ 1.333, $\Phi_{S T}=0.79 ; \eta_{X}=1.445(\mathbf{B B F}$ and $\mathbf{2 B F})$ and $1.431(\mathbf{3})$, and Grad the gradient from the plot of integrated fluorescence intensity vs absorbance. ${ }^{1}$

X-ray intensity data were measured on a Bruker CCD-based and PHOTON II CPAD-based diffractometer with dual $\mathrm{Cu} / \mathrm{Mo}$ ImuS microfocus optics $(\mathrm{Cu} \mathrm{K} \alpha$ radiation, $\lambda=1.54178 \AA$, Mo $\mathrm{K} \alpha$ radiation, $\lambda=0.71073 \AA$ ). Crystals were mounted on a cryoloop using Paratone oil and placed under a steam of nitrogen at $100 \mathrm{~K}$ (Oxford Cryosystems). The detector was placed at a distance of $5.00 \mathrm{~cm}$ from the crystal. The data were corrected for absorption with the SADABS program. 
The structures were refined using the Bruker SHELXTL Software Package (Version 6.1), ${ }^{2}$ and were solved using direct methods until the final anisotropic full-matrix, least squares refinement of $\mathrm{F}^{2}$ converged. The disordered toluene solvent in structure 1BF was squeezed out by PLATON. ${ }^{3}$

Electrochemistry measurements were conducted using a CHI 820D potentiostat in a standard three-electrode configuration. Platinum wire was used as the counter electrode. The working electrode used was a $2 \mathrm{~mm}$ diameter platinum disk. A nonaqueous $\mathrm{Ag} / \mathrm{Ag}^{+}$reference electrode was used by immersing silver wire in a degassed DMF solution of $0.01 \mathrm{M} \mathrm{AgNO}_{3} / 0.1$ $\mathrm{M}$ tetrabutylammonium hexafluorophosphate $\left(\mathrm{TBAPF}_{6}\right)$. All potentials were referenced to the ferrocene/ferrocenium couple. The concentration of analyte was $1.0 \mathrm{mM}$, and the supporting electrolyte was $0.1 \mathrm{M} \mathrm{TBAPF}_{6}$ dissolved in DMF.

\section{Computational Details}

The starting geometries of compounds 1, 2, 1BF, and 2BF were optimized using a B3LYP exchange-correlation functional. ${ }^{4}$ Energy minima in optimized geometry were confirmed by the frequency calculations (absence of the imaginary frequencies). The solvent effect was modeled using the polarized continuum model (PCM). ${ }^{5}$ In all calculations, DMF was used as the solvent. In PCM-TDDFT calculation, the first 50 states were calculated. All atoms were modeled using the 6-311G(d $)^{6}$ basis set. Gaussian 09 software was used in all calculations. ${ }^{7}$ The QMForge program was used for molecular orbital analysis in all cases. ${ }^{8}$ 


\section{Full citation for Gaussian}

Gaussian 09, Revision D.01,. Frisch, M. J; Trucks, G. W.; Schlegel, H. B.; Scuseria, G. E.; Robb, M. A.; Cheeseman, J. R.; Montgomery, Jr., J. A.; Vreven, T.; Kudin, K. N.; Burant, J. C.; Millam, J. M.; Iyengar, S. S.; Tomasi, J.; Barone, V.; Mennucci, B.; Cossi, M.; Scalmani, G.; Rega, N.; Petersson, G. A.; Nakatsuji, H.; Hada, M.; Ehara, M.; Toyota, K.; Fukuda, R.; Hasegawa, J.; Ishida, M.; Nakajima, T.; Honda, Y.; Kitao, O.; Nakai, H.; Klene, M.; Li, X.; Knox, J. E.; Hratchian, H. P.; Cross, J. B.; Adamo, C.; Jaramillo, J.; Gomperts, R.; Stratmann, R. E.; Yazyev, O.; Austin, A. J.; Cammi, R.; Pomelli, C.; Ochterski, J. W.; Ayala, P. Y.; Morokuma, K.; Voth, G. A.; Salvador, P.; Dannenberg, J. J.;. Zakrzewski, V. G.; Dapprich, S.; Daniels, A. D.; Strain, M. C.; Farkas, O.; Malick, D. K.; Rabuck, A. D.; Raghavachari, K.; Foresman, J. B.; Ortiz, J. V.; Cui, Q.; Baboul, A. G.; Clifford, S.;. Cioslowski, J.; Stefanov, B. B.; Liu, G.;. Liashenko, A.; Piskorz, P.; Komaromi, I.; Martin, R. L.; Fox, D. J.; Keith, T.; Al-Laham, M. A.; Peng, C. Y.; Nanayakkara, A.; Challacombe, M.; Gill, P. M. W.; Johnson, B.; Chen, W.; Wong, M. W.; Gonzalez, C.; Pople, J. A. Gaussian, Inc., Wallingford CT, 2009. 
Synthesis of $\mathbf{1}$ and 2 . The procedure for generating $\mathbf{1}$ is the same as $\mathbf{2}$ except $1 \mathrm{H}$-indazole-3-amine (2.08 g, $15.61 \mathrm{mmol})$ was used in 2. Phthalonitrile $(1.00 \mathrm{~g}, 7.80 \mathrm{mmol})$ and 3-amino pyrazole $\left(1.30 \mathrm{~g}, 15.61 \mathrm{mmol}\right.$ were mixed together and heated on a sand bath at $180{ }^{\circ} \mathrm{C}$ for 1 hour. The residue was cooled to room temperature, and dissolved in a minimal amount of DMF. DI water was added to induce precipitation of the product. The resultant solid was filtered and air dried to give a yellow powder. Crystals of $\mathbf{1}$ and $\mathbf{2}$ suitable for X-ray diffraction were grown from slow evaporation from DMF.
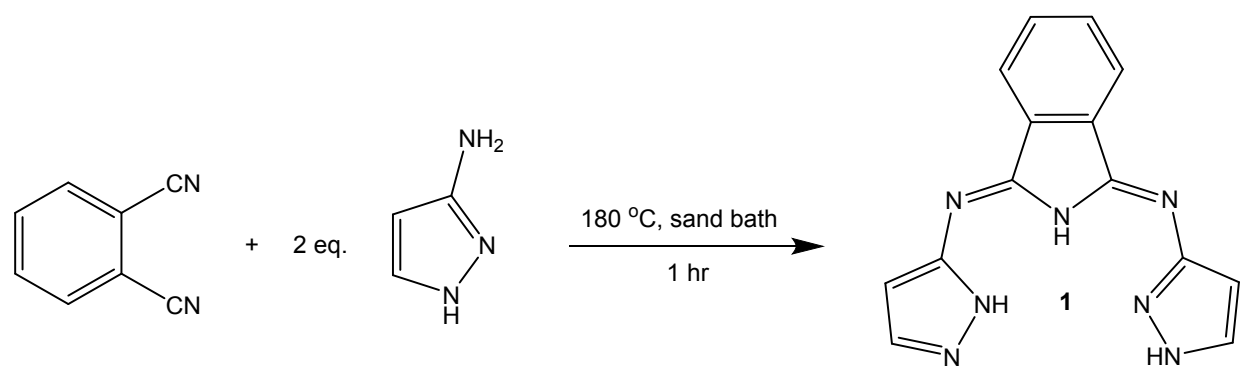

1: Yield: $1.56 \mathrm{~g}$ (72\%). MP: $240-247^{\circ} \mathrm{C}$. IR: $3132 \mathrm{~cm}^{-1}\left(v_{\mathrm{NH}}\right), 1632 \mathrm{~cm}^{-1}\left(v_{\mathrm{CN}(\text { imine) }}\right)$. ${ }^{1} \mathrm{H}$ NMR (300 MHz, $\left.\mathrm{d}_{6}-\mathrm{DMSO}\right): \delta=12.79(\mathrm{~s}, 2 \mathrm{H}, \mathrm{NH}), 11.91(\mathrm{~s}, 1 \mathrm{H}, \mathrm{NH}), 7.93(\mathrm{~m}, 2 \mathrm{H}, \mathrm{H}$ on isoindoline), 7.77 (s, $2 \mathrm{H}, \mathrm{H}$ on pyrazole), 7.68 (m, $2 \mathrm{H}, \mathrm{H}$ on isoindoline), 6.33 (s, $2 \mathrm{H}, \mathrm{H}$ on pyrazole). ${ }^{13} \mathrm{C}\left\{{ }^{1} \mathrm{H}\right\}$ NMR (125 MHz, $\left.\mathrm{d}_{6}-\mathrm{DMSO}\right): \delta=155.6,148.3,134.9,131.4,129.8,121.8,102.6$. HRMS (ESITOF, positive mode) $\mathrm{m} / \mathrm{z}$ : calcd for $\mathrm{C}_{14} \mathrm{H}_{12} \mathrm{~N}_{7} 278.1149$, found $278.1151[\mathrm{M}+\mathrm{H}]^{+}$. 

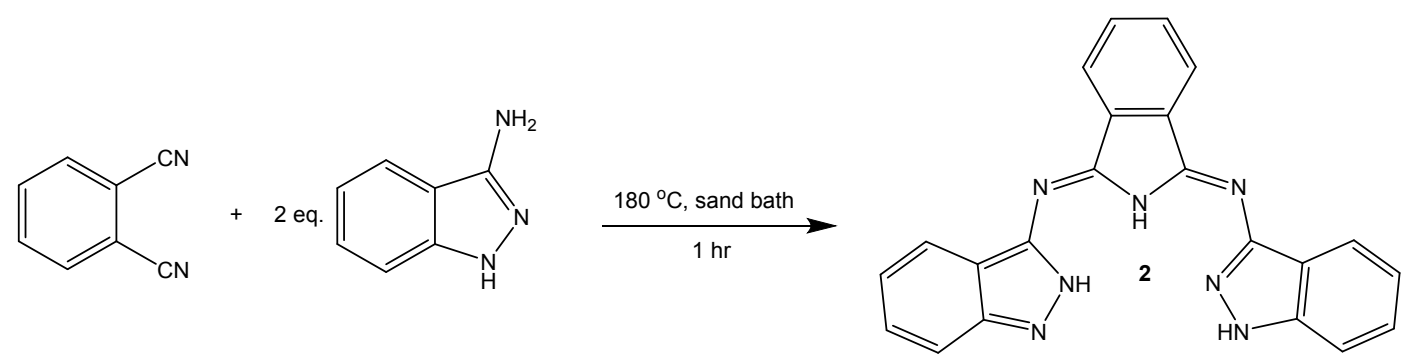

2: Yield: $2.24 \mathrm{~g}$ (76\%). MP: $307-312{ }^{\circ} \mathrm{C}$. IR: $3183 \mathrm{~cm}^{-1}\left(v_{\mathrm{NH}}\right), 1635 \mathrm{~cm}^{-1}\left(v_{\mathrm{CN}(\mathrm{imine})}\right)$. ${ }^{1} \mathrm{H}$ NMR (300 MHz, $\left.\mathrm{d}_{6}-\mathrm{DMSO}\right): \delta=13.13(\mathrm{~s}, 2 \mathrm{H}, \mathrm{NH}), 12.33(\mathrm{~s}, 1 \mathrm{H}, \mathrm{NH}), 8.13(\mathrm{~m}, 2 \mathrm{H}, \mathrm{H}$ on isoindoline), $7.97(\mathrm{~d}, \mathrm{~J}=8.20 \mathrm{~Hz}, 2 \mathrm{H}, \mathrm{H}$ on indazole), $7.78(\mathrm{~m}, 2 \mathrm{H}, \mathrm{H}$ on isoindoline), $7.57(\mathrm{~d}, \mathrm{~J}=8.49 \mathrm{~Hz}, 2 \mathrm{H}$, $\mathrm{H}$ on indazole), $7.46(\mathrm{t}, \mathrm{J}=7.90 \mathrm{~Hz}, 2 \mathrm{H}, \mathrm{H}$ on indazole), $7.24(\mathrm{t}, \mathrm{J}=7.90 \mathrm{~Hz}, 2 \mathrm{H}, \mathrm{H}$ on indazole). ${ }^{13} \mathrm{C}\left\{{ }^{1} \mathrm{H}\right\}$ NMR (125 MHz, $\left.\mathrm{d}_{6}-\mathrm{DMSO}\right): \delta=149.4,148.9,140.9,134.8,134.0,133.9$, 131.6, 127.0, 122.1, 120.7, 119.9, 119.8, 110.4. HRMS (ESI-TOF, positive mode) m/z: calcd for $\mathrm{C}_{22} \mathrm{H}_{16} \mathrm{~N}_{7} 378.1462$, found $378.1460[\mathrm{M}+\mathrm{H}]^{+}$.

Synthesis of $1 \mathrm{BF}$ and $2 \mathrm{BF}$. The procedure for generating $1 \mathrm{BF}$ is the same as $2 \mathrm{BF}$ except ligand $2(1.36 \mathrm{~g}, 3.6 \mathrm{mmol})$ was used in $\mathbf{2 B F}$ and dry DMF was used as the solvent. Triethylamine (10 $\mathrm{mL}, 36.1 \mathrm{mmol})$, and $\mathrm{BF}_{3} \cdot \mathrm{OEt}_{2}(3.2 \mathrm{~mL}, 25.2 \mathrm{mmol})$ were added to ligand $\mathbf{1}(1.00 \mathrm{~g}, 3.6 \mathrm{mmol})$ in dry toluene $(15 \mathrm{~mL})$. The reaction was refluxed overnight under $\mathrm{N}_{2}$. The solution was then cooled to room temperature and DI water was added to induce precipitation of the product. The crude materials was recrystallized in toluene to yield crystals of pure material that were filtered and rinsed with cold toluene, to give a yellow solid (red solid for $\mathbf{2 B F}$ ). Crystals of $\mathbf{1 B F}$ and $\mathbf{2 B F}$ suitable for X-ray diffraction were grown from toluene. 

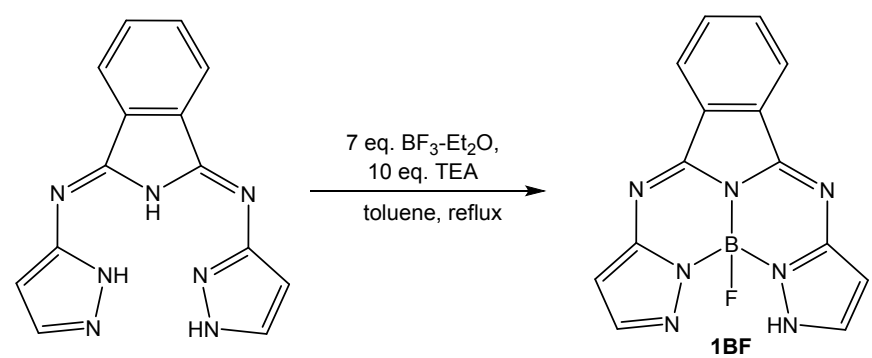

1BF: Yield: $0.59 \mathrm{~g}(54 \%)$. IR: $1601 \mathrm{~cm}^{-1}\left(v_{\mathrm{CN}(\mathrm{imine})}\right) .{ }^{1} \mathrm{H} \mathrm{NMR}\left(300 \mathrm{MHz}, \mathrm{CDCl}_{3}\right): \delta=12.53(\mathrm{~s}$, $\mathrm{NH}), 7.96(\mathrm{~m}, 2 \mathrm{H}, \mathrm{H}$ on isoindoline), 7.56-7.53 (m, 4H, H on isoindoline and pyrazole), 6.32 (s, $2 \mathrm{H}, \mathrm{H}$ on pyrazole $) .{ }^{13} \mathrm{C}\left\{{ }^{1} \mathrm{H}\right\} \mathrm{NMR}\left(125 \mathrm{MHz}, \mathrm{d}_{6}-\mathrm{DMSO}\right): \delta=155.8,151.0,135.3,131.1,129.7$, 128.9, 122.3, 122.1, 102.5. ${ }^{19} \mathrm{~F}\left(282 \mathrm{MHz}, \mathrm{CDCl}_{3}\right): \delta=-149.93$. HRMS (ESI-TOF, negative mode) $\mathrm{m} / \mathrm{z}$ : calcd for $\mathrm{C}_{14} \mathrm{H}_{8} \mathrm{BFN}_{7}$ 304.0924, found 304.0918 [M-H]-
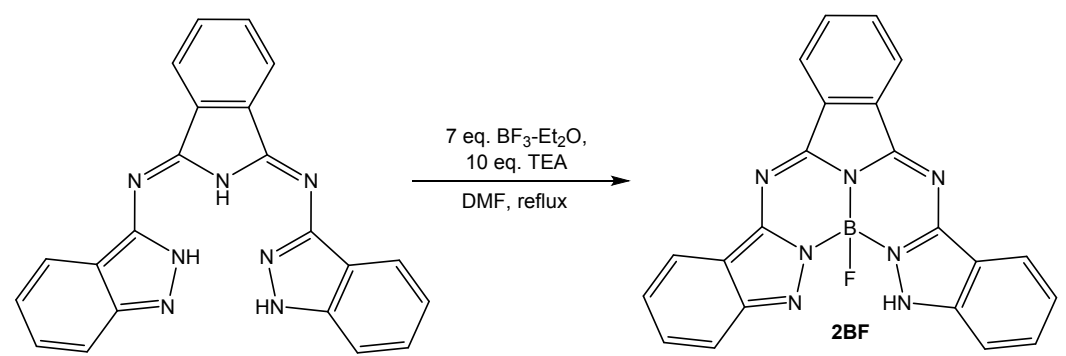

2BF: Yield: $0.98 \mathrm{~g}(67 \%)$. IR: $1569 \mathrm{~cm}^{-1}\left(v_{\mathrm{CN}(\mathrm{imine})}\right) .{ }^{1} \mathrm{H} \mathrm{NMR}\left(300 \mathrm{MHz}, \mathrm{CDCl}_{3}\right): \delta=13.87(\mathrm{~s}$, $\mathrm{NH}), 8.24(\mathrm{~m}, 2 \mathrm{H}, \mathrm{H}$ on isoindoline $), 8.19(\mathrm{~d}, \mathrm{~J}=8.25 \mathrm{~Hz}, 2 \mathrm{H}, \mathrm{H}$ on indazole $), 7.74(\mathrm{~m}, 2 \mathrm{H}, \mathrm{H}$ on isoindoline), $7.63(\mathrm{~d}, \mathrm{~J}=8.46 \mathrm{~Hz}, 2 \mathrm{H}, \mathrm{H}$ on indazole), $7.50(\mathrm{t}, \mathrm{J}=7.25 \mathrm{~Hz}, 2 \mathrm{H}, \mathrm{H}$ on indazole), $7.29\left(\mathrm{t}, \mathrm{J}=7.25 \mathrm{~Hz}, 2 \mathrm{H}, \mathrm{H}\right.$ on indazole). ${ }^{13} \mathrm{C}\left\{{ }^{1} \mathrm{H}\right\} \mathrm{NMR}\left(125 \mathrm{MHz}, \mathrm{CDCl}_{3}\right): \delta=151.2,150.5$, 141.0, 134.9, 130.9, 126.6, 122.6, 122.3, 121.1, 120.5, 120.4, 117.6, 116.0, 110.1. ${ }^{19} \mathrm{~F}(282 \mathrm{MHz}$ $\mathrm{CDCl}_{3}$ ): $\delta=-149.22$. HRMS (ESI-TOF, negative mode) $\mathrm{m} / \mathrm{z}$ : calcd for $\mathrm{C}_{22} \mathrm{H}_{12} \mathrm{BFN}_{7} 404.1237$, found 404. $1234[\mathrm{M}-\mathrm{H}]^{-}$. 
Synthesis of 3. Ligand $2(0.25 \mathrm{~g}, 0.66 \mathrm{mmol})$ was combined with $\mathrm{BF}_{3} \cdot \mathrm{OEt}_{2}(1 \mathrm{~mL})$ in a round bottom flask (no base) and heated to $100{ }^{\circ} \mathrm{C}$ for $1 \mathrm{hr}$. During this time the initial deep red solution eventually yielded a tan precipitate. The solution was brought to room temperature and the solids were filtered and washed with water. The crude material was recrystallized in acetone to yield crystals of pure material that were filtered and rinsed with cold acetone. Crystals suitable for Xray diffraction were grown from acetone.
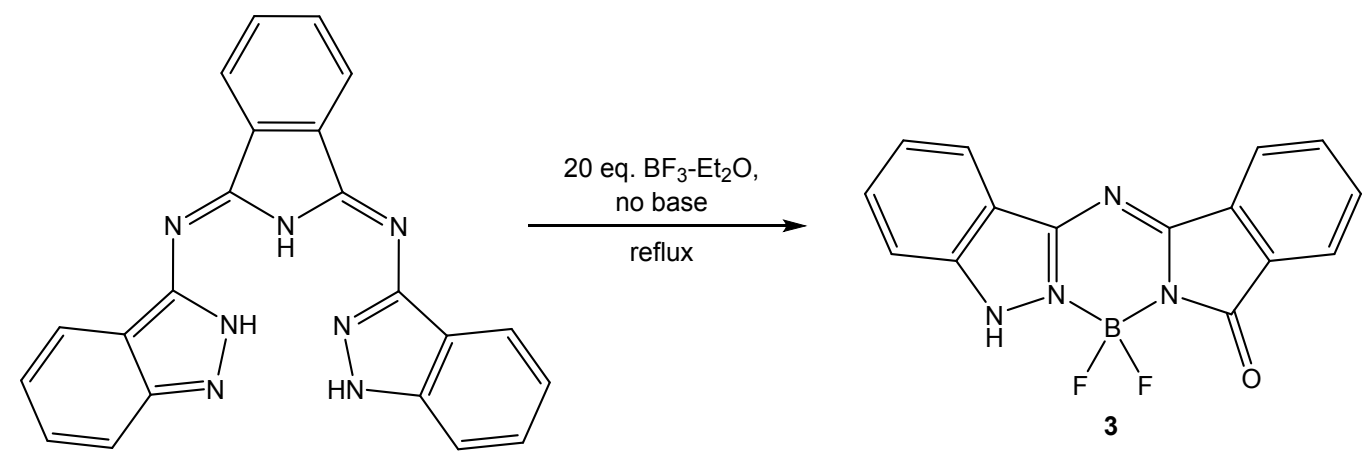

3: Yield: $0.11 \mathrm{~g}$ (53\%). IR: $1723 \mathrm{~cm}^{-1}\left(v_{\mathrm{CO}}\right) .{ }^{1} \mathrm{H}$ NMR (300 MHz, $\left.\mathrm{d}_{6}-\mathrm{DMSO}\right): \delta=8.13(\mathrm{~d}, \mathrm{~J}=$ $8.25 \mathrm{~Hz}, 1 \mathrm{H}), 8.08(\mathrm{~d}, \mathrm{~J}=7.05 \mathrm{~Hz}, 1 \mathrm{H}), 7.93-7.75(\mathrm{~m}, 4 \mathrm{H}), 7.64(\mathrm{~d}, \mathrm{~J}=8.66 \mathrm{~Hz}, 1 \mathrm{H}, \mathrm{H}$ on indazole $)$, $7.45\left(\mathrm{t}, \mathrm{J}=7.45 \mathrm{~Hz}, 1 \mathrm{H}, \mathrm{H}\right.$ on indazole). ${ }^{13} \mathrm{C}\left\{{ }^{1} \mathrm{H}\right\} \mathrm{NMR}\left(125 \mathrm{MHz}, \mathrm{d}_{6}-\mathrm{DMSO}\right): \delta=170.5,158.8$, $144.9,141.0,135.4,134.3,133.6,132.4,131.2,123.8,123.6,122.8,121.4,115.3,111.6 .{ }^{19} \mathrm{~F}(282$ $\mathrm{MHz}, \mathrm{CDCl}_{3}$ ): $\delta=-144.65,144.59$. HRMS (ESI-TOF, negative mode) $\mathrm{m} / \mathrm{z}$ : calcd for $\mathrm{C}_{15} \mathrm{H}_{8} \mathrm{BF}_{2} \mathrm{~N}_{4} \mathrm{O}$ 309.0765, found 309.0757 [M-H]'. 

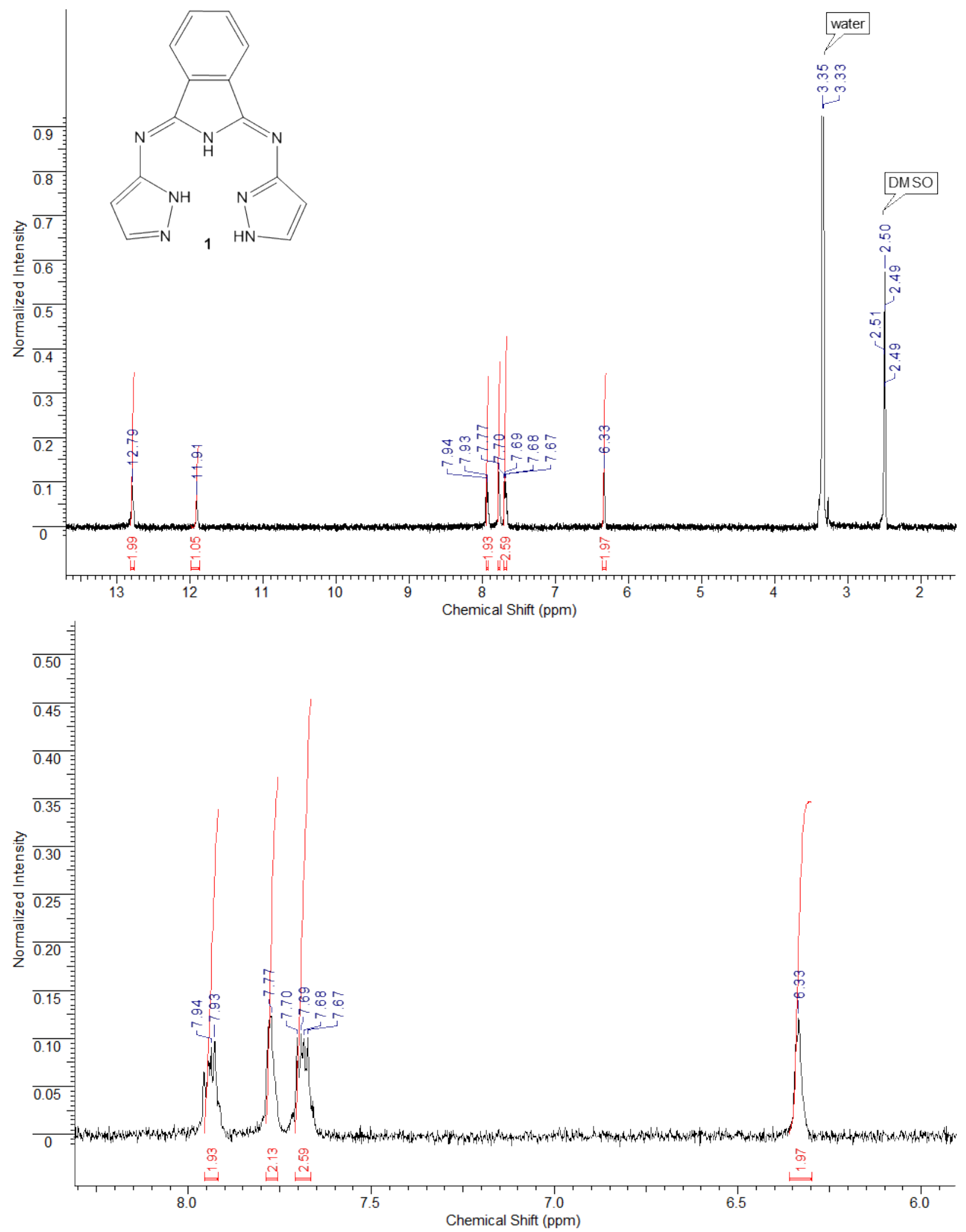

Figure S1: ${ }^{1} \mathrm{H}$ NMR (300 MHz) of 1 in d6-DMSO. 

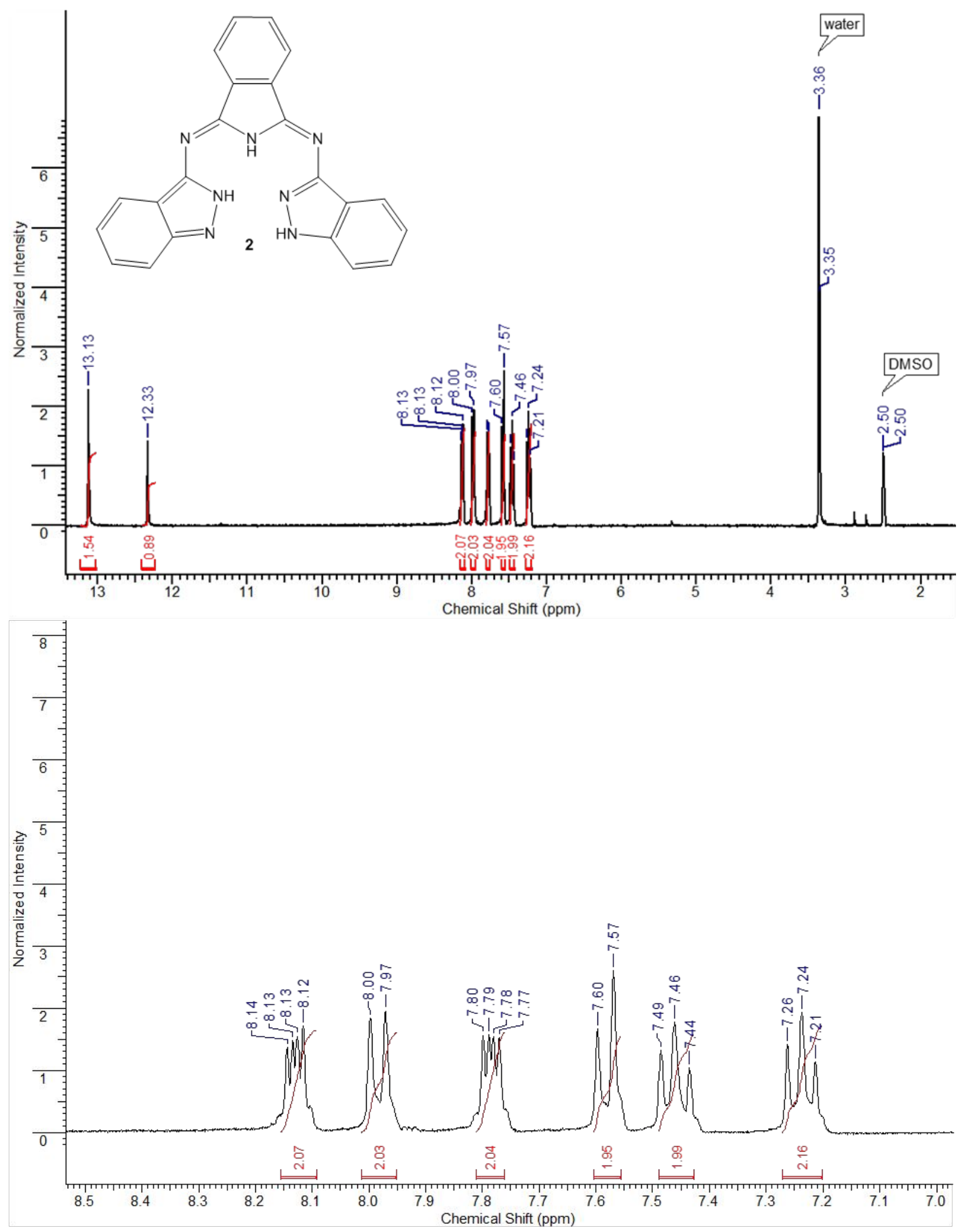

Figure S2: ${ }^{1} \mathrm{H}$ NMR $(300 \mathrm{MHz})$ of 2 in d6-DMSO. 


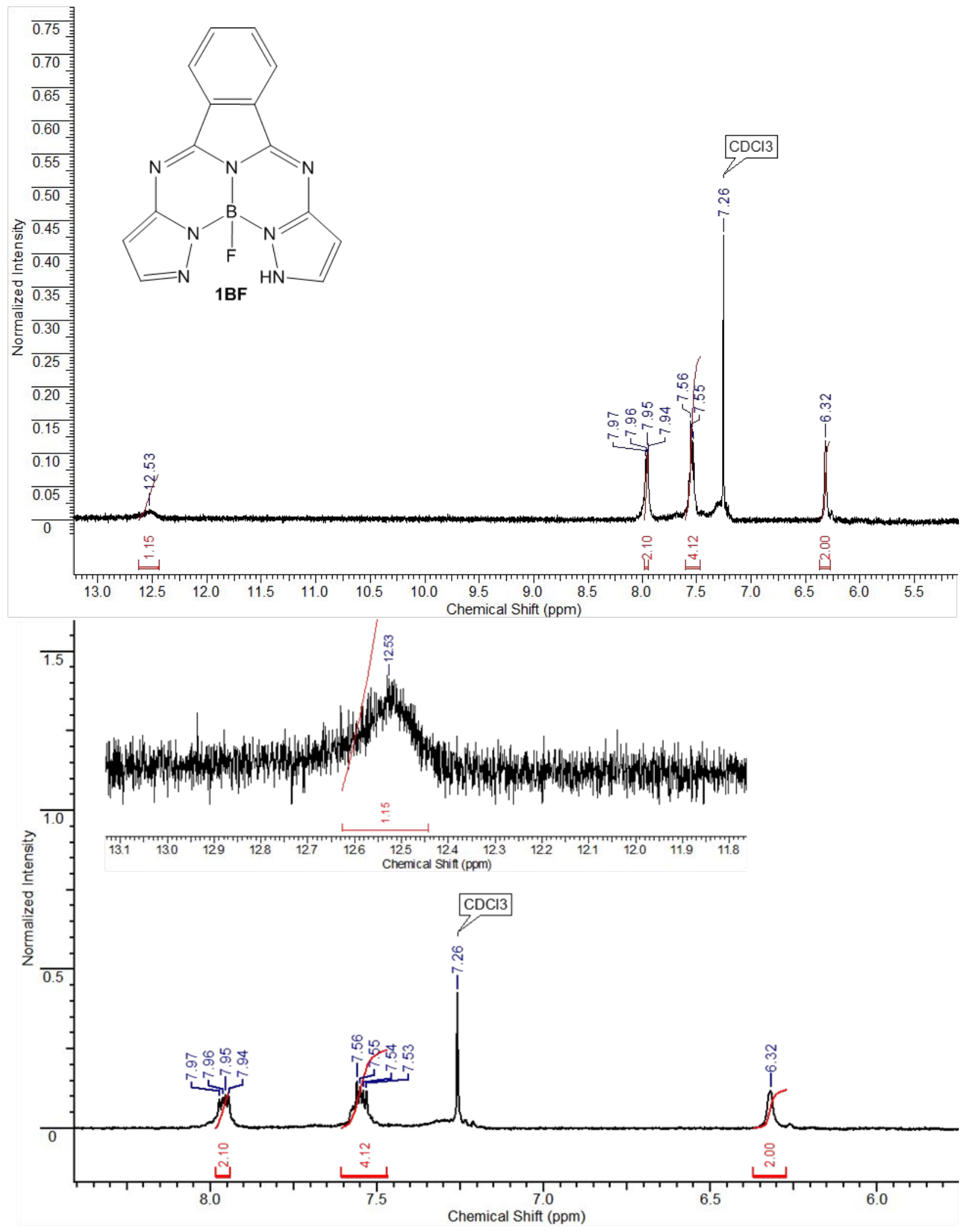

Figure S3: ${ }^{1} \mathrm{H}$ NMR (300 MHz) of $\mathbf{1 B F}$ in $\mathrm{CDCl}_{3}$. 

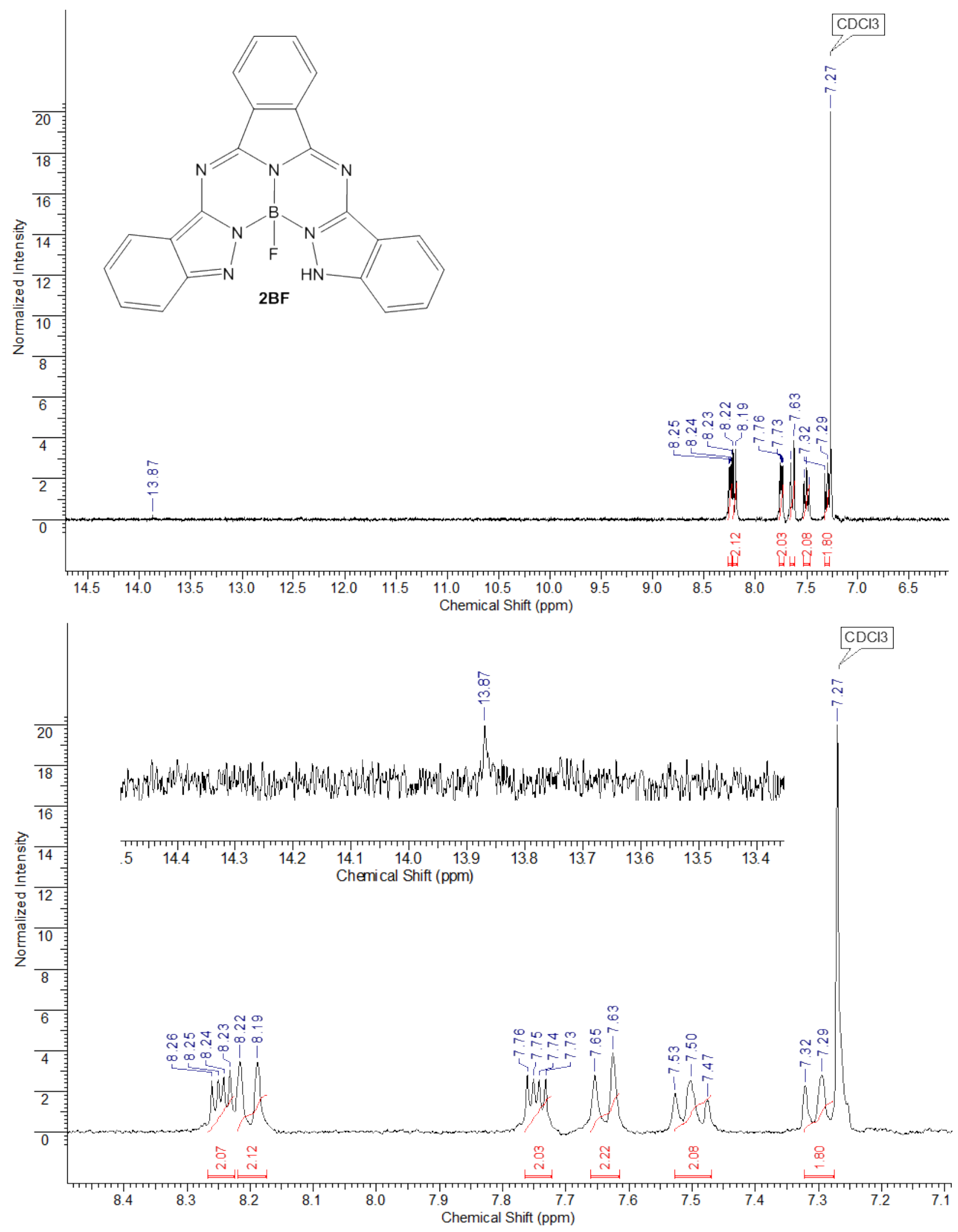

Figure S4: ${ }^{1} \mathrm{H}$ NMR $(300 \mathrm{MHz})$ of $\mathbf{2 B F}$ in $\mathrm{CDCl}_{3}$. 

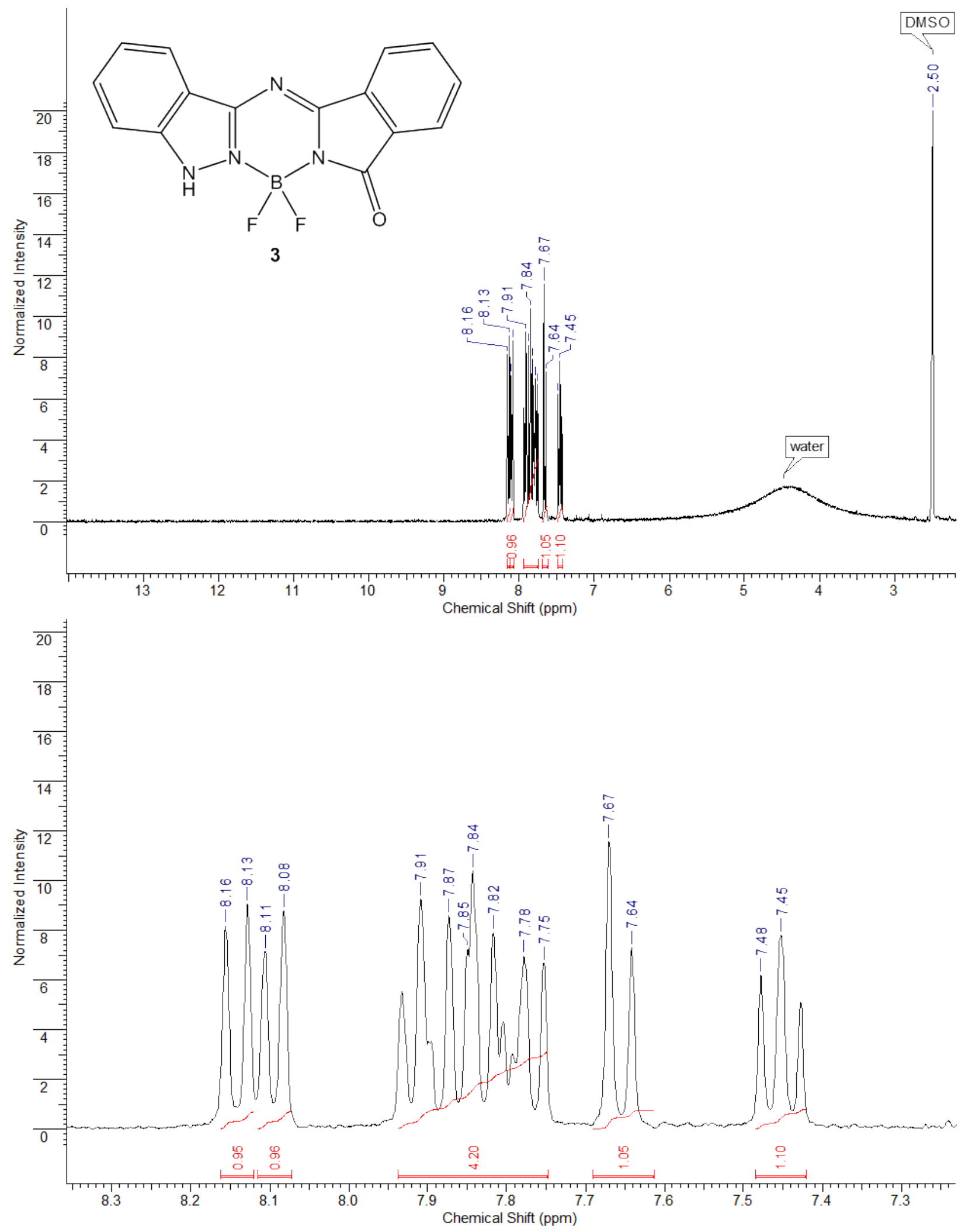

Figure S5: ${ }^{1} \mathrm{H}$ NMR (300 MHz) of 3 in d6-DMSO. 


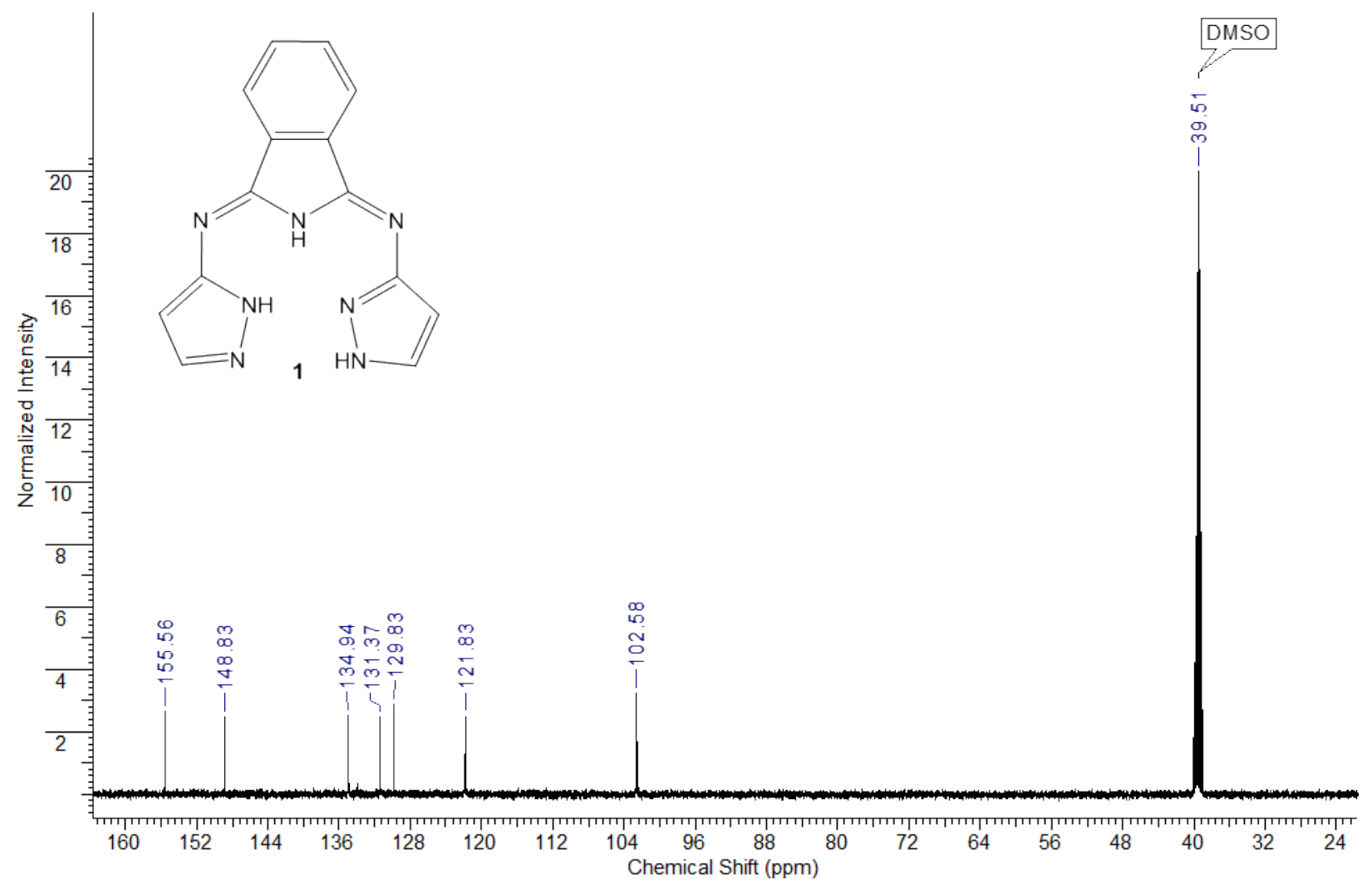

Figure S6: ${ }^{13} \mathrm{C}\left\{{ }^{1} \mathrm{H}\right\}$ NMR $(125 \mathrm{MHz})$ of $\mathbf{1}$ in d6-DMSO. 


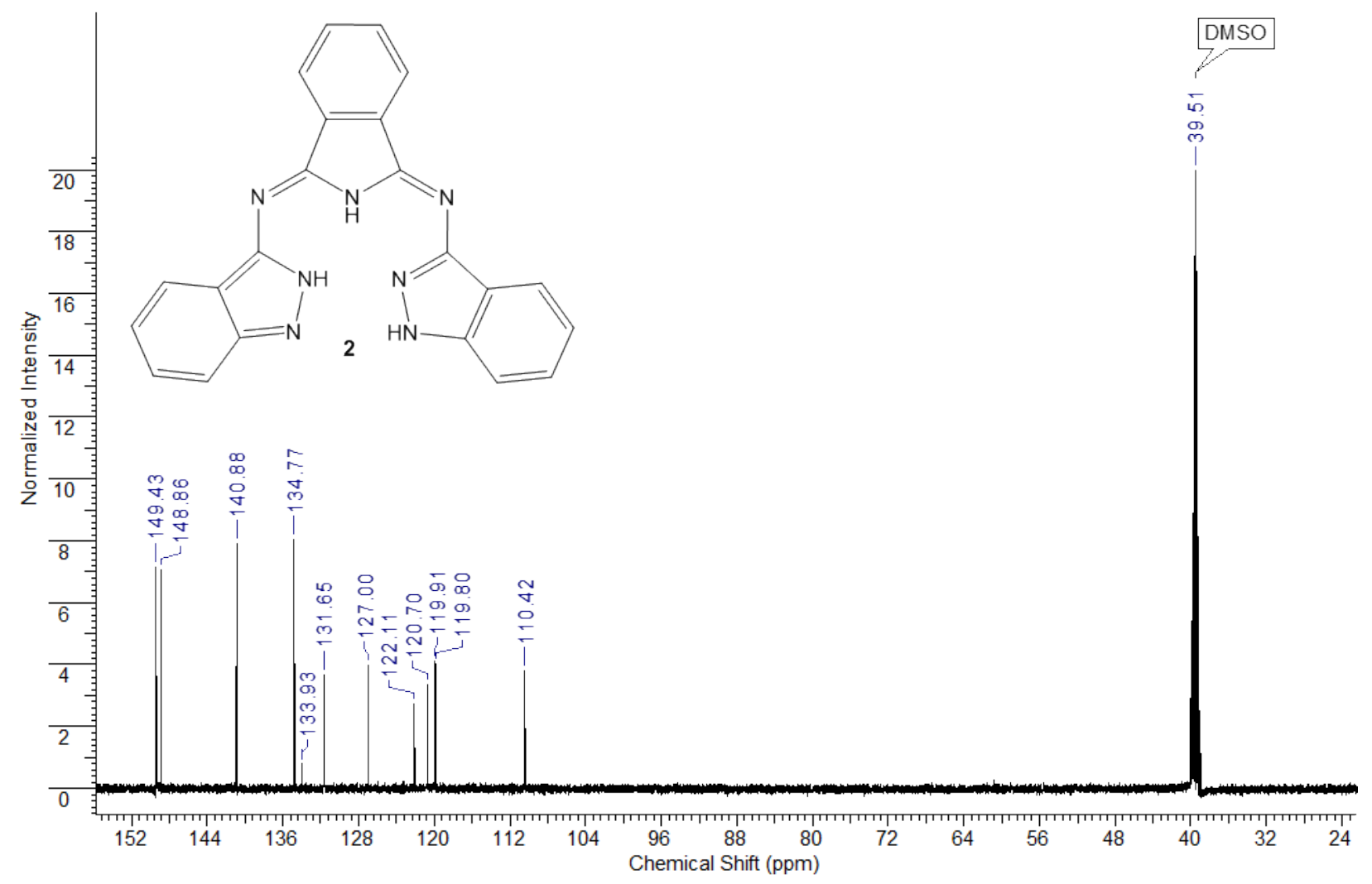

Figure S7: ${ }^{13} \mathrm{C}\left\{{ }^{1} \mathrm{H}\right\}$ NMR $(125 \mathrm{MHz})$ of 2 in d6-DMSO. 


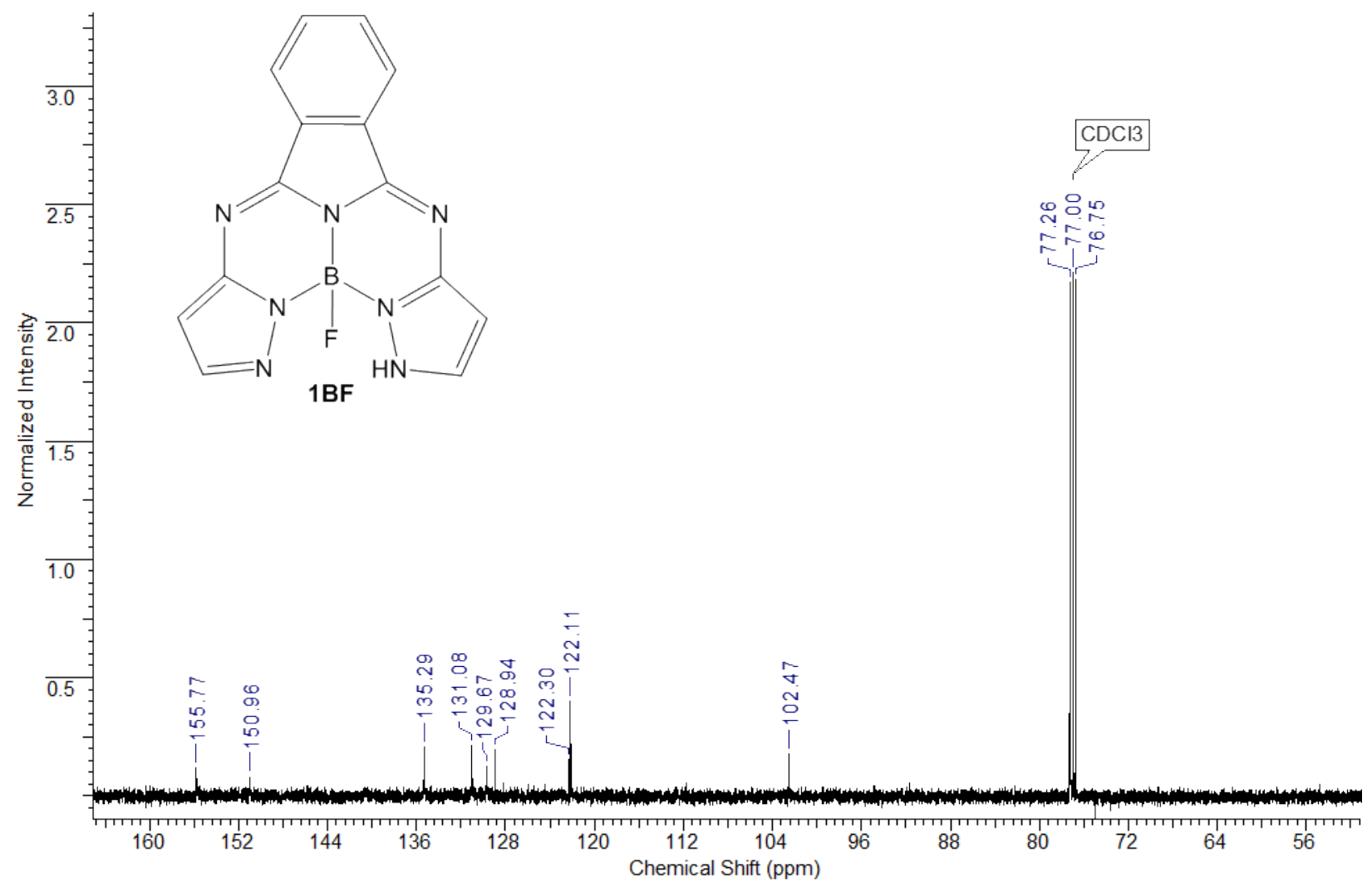

Figure S8: ${ }^{13} \mathrm{C}\left\{{ }^{1} \mathrm{H}\right\}$ NMR $(125 \mathrm{MHz})$ of $\mathbf{1 B F}$ in $\mathrm{CDCl}_{3}$. 


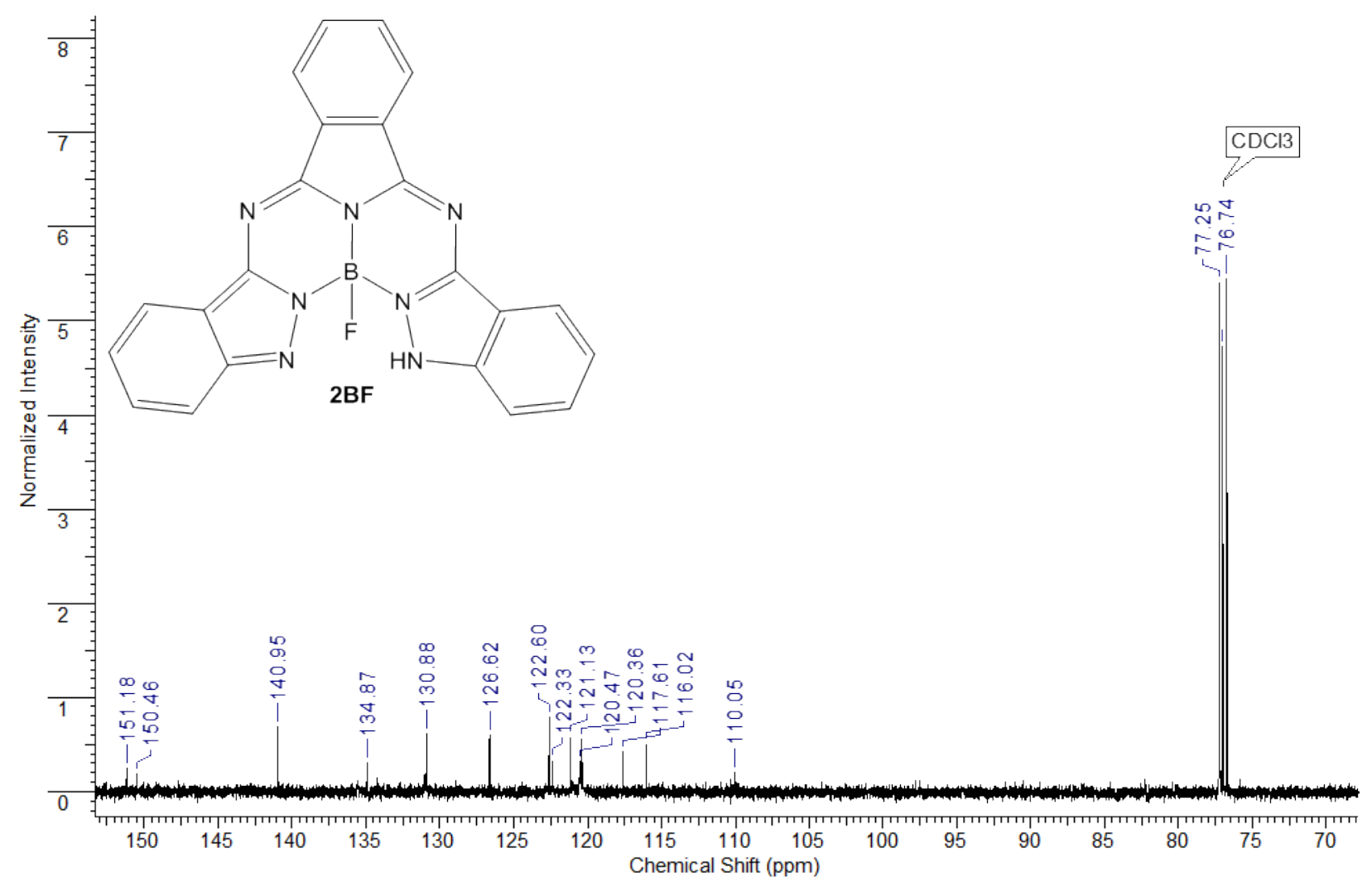

Figure S9: ${ }^{13} \mathrm{C}\left\{{ }^{1} \mathrm{H}\right\} \mathrm{NMR}(125 \mathrm{MHz})$ of $\mathbf{2 B F}$ in $\mathrm{CDCl}_{3}$. 


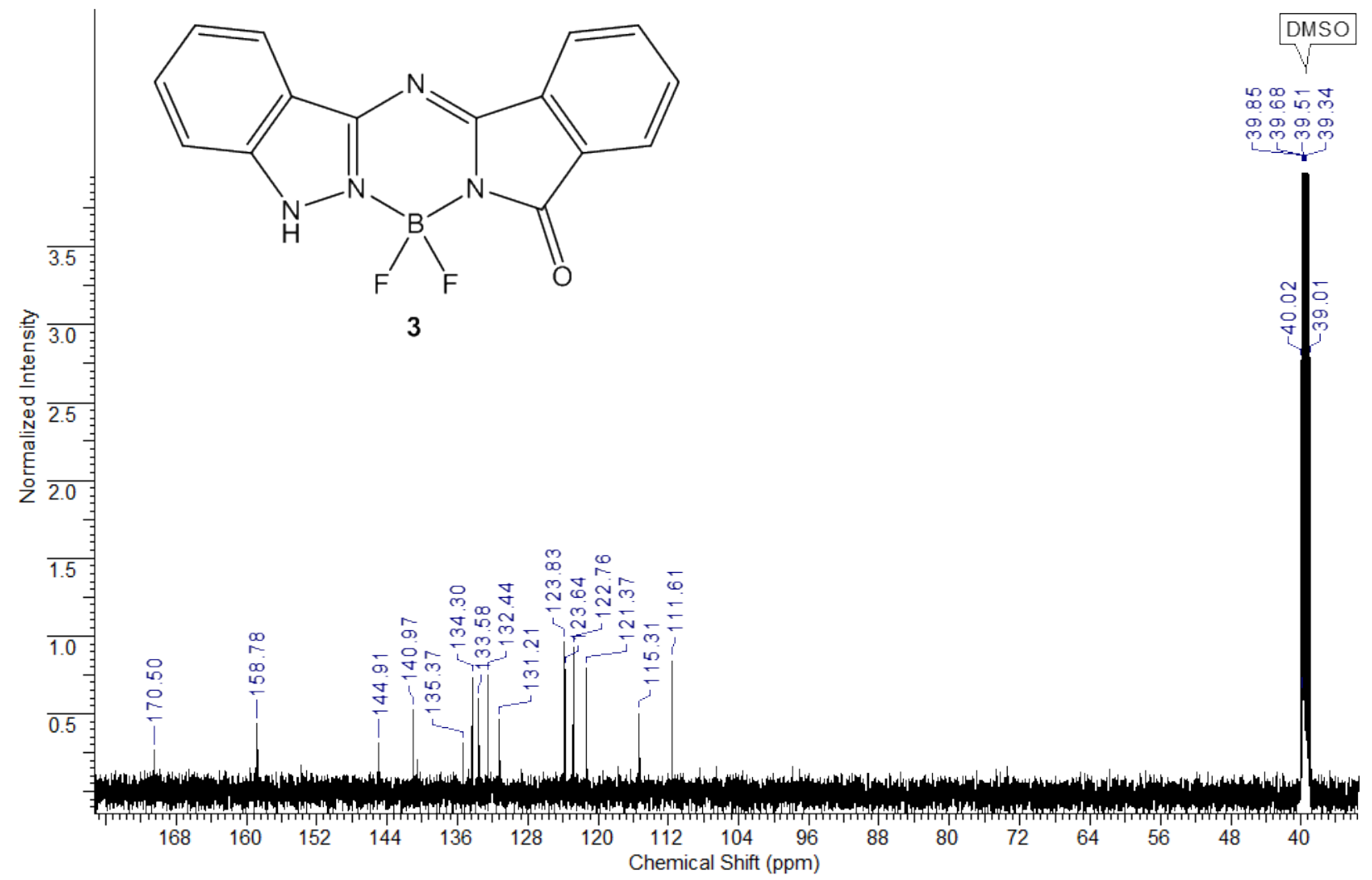

Figure S10: ${ }^{13} \mathrm{C}\left\{{ }^{1} \mathrm{H}\right\}$ NMR $(125 \mathrm{MHz})$ of $\mathbf{3}$ in d6-DMSO. 


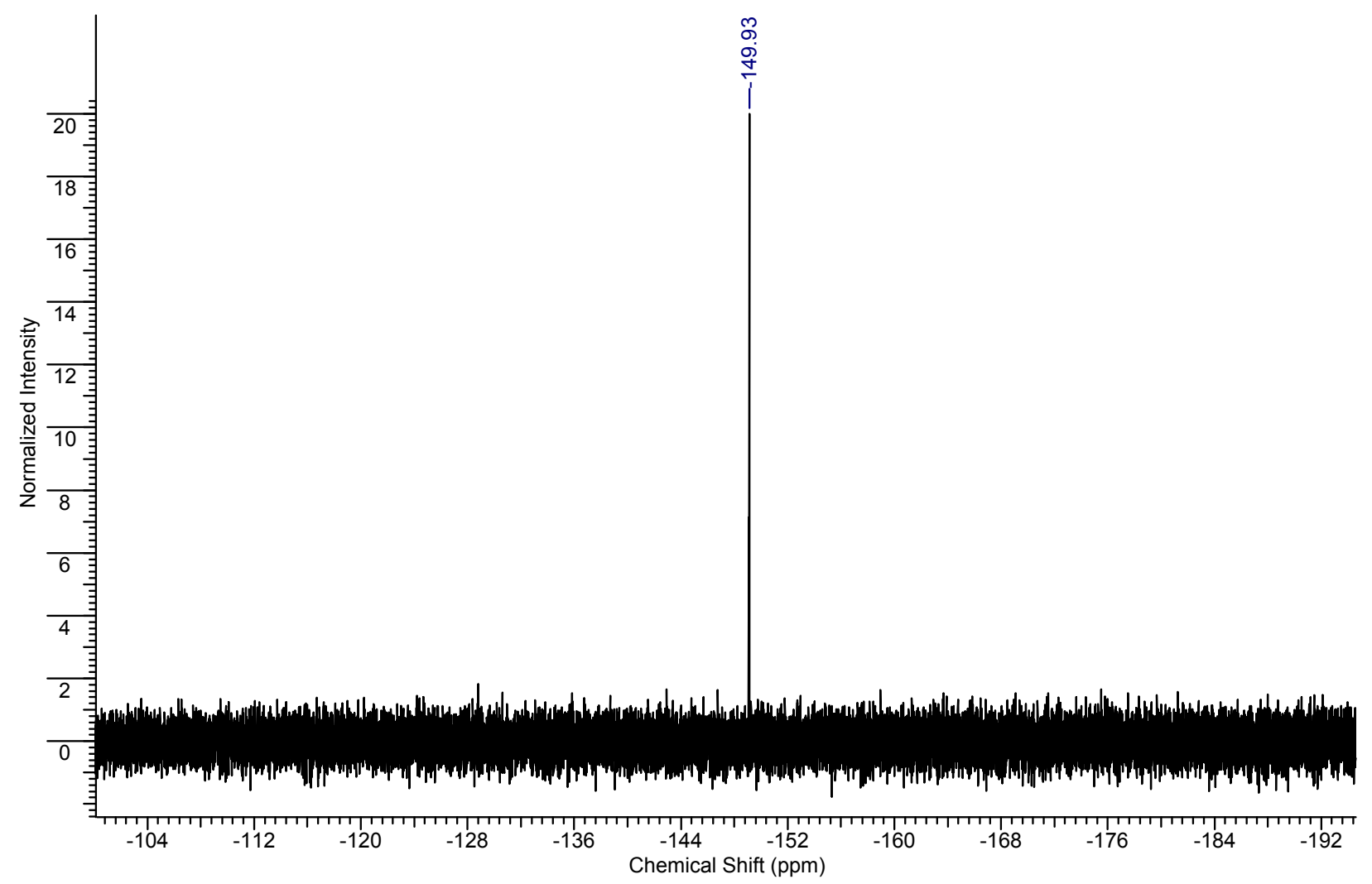

Figure S11: ${ }^{19} \mathrm{~F}$ NMR (282 MHz) of $\mathbf{1 B F}$ in $\mathrm{CDCl}_{3}$. 


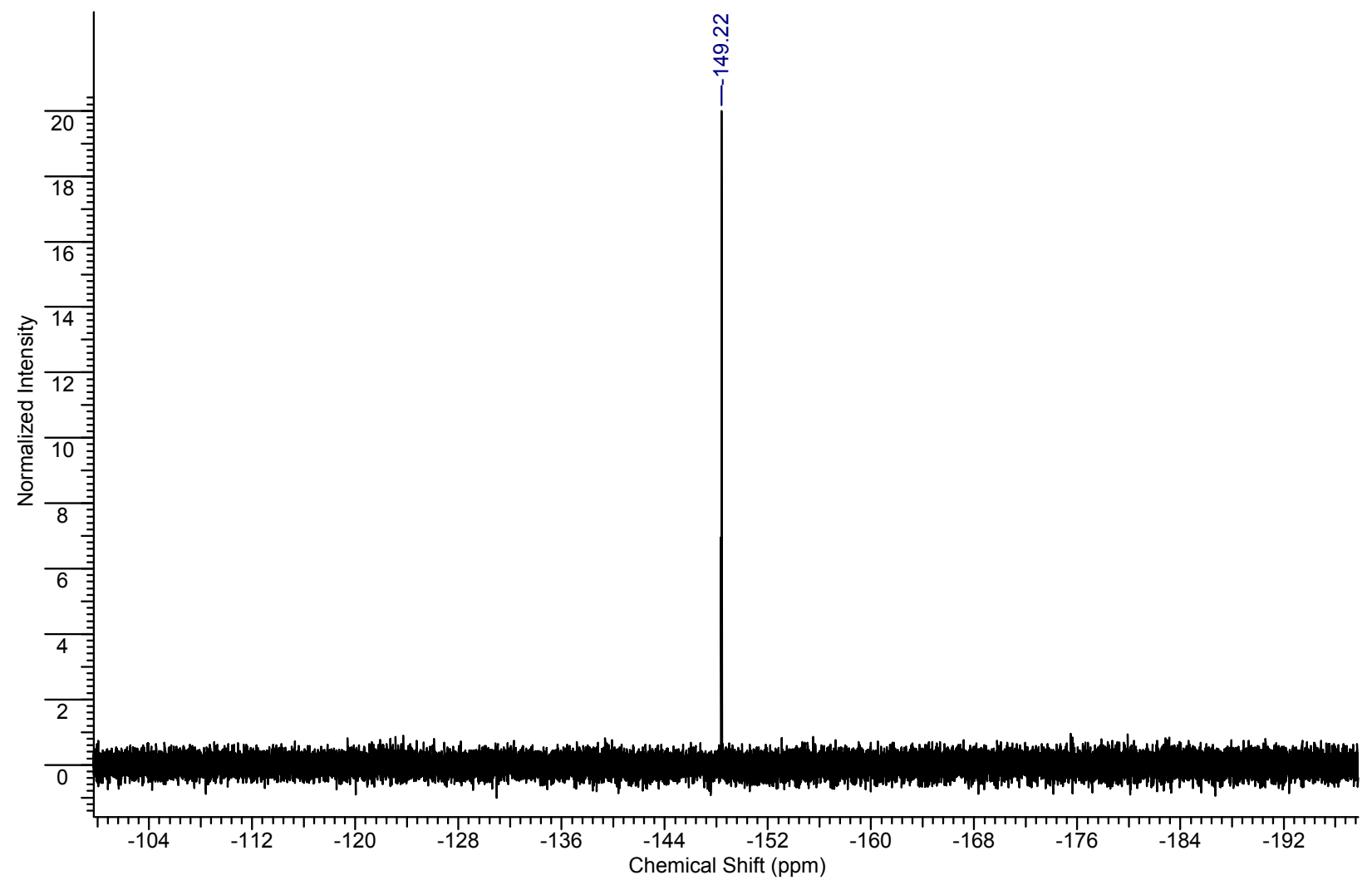

Figure S12: ${ }^{19} \mathrm{~F}$ NMR (282 MHz) of $\mathbf{2 B F}$ in $\mathrm{CDCl}_{3}$. 


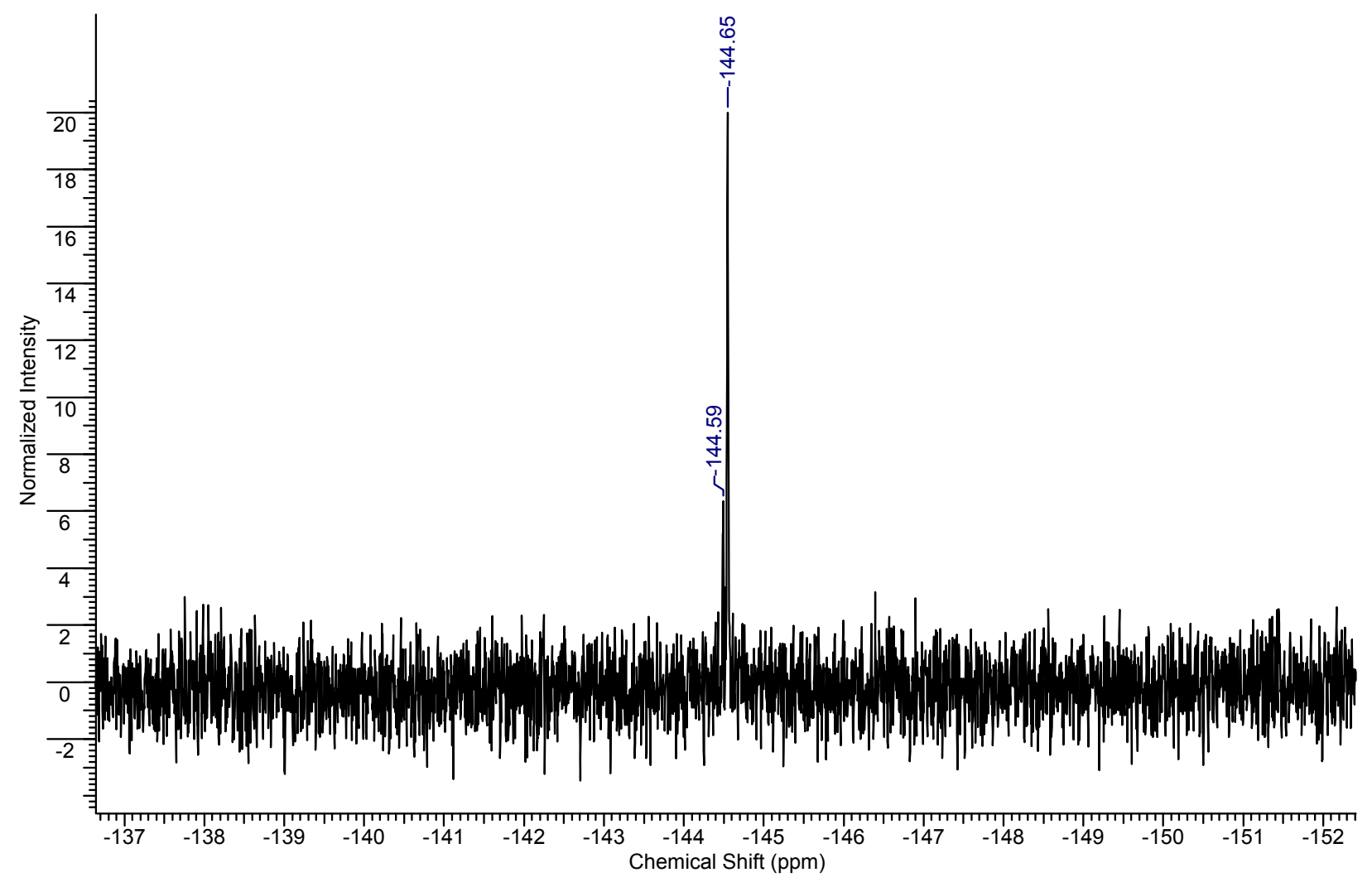

Figure S13: ${ }^{19} \mathrm{~F}$ NMR $(282 \mathrm{MHz})$ of 3 in d6-DMSO. 

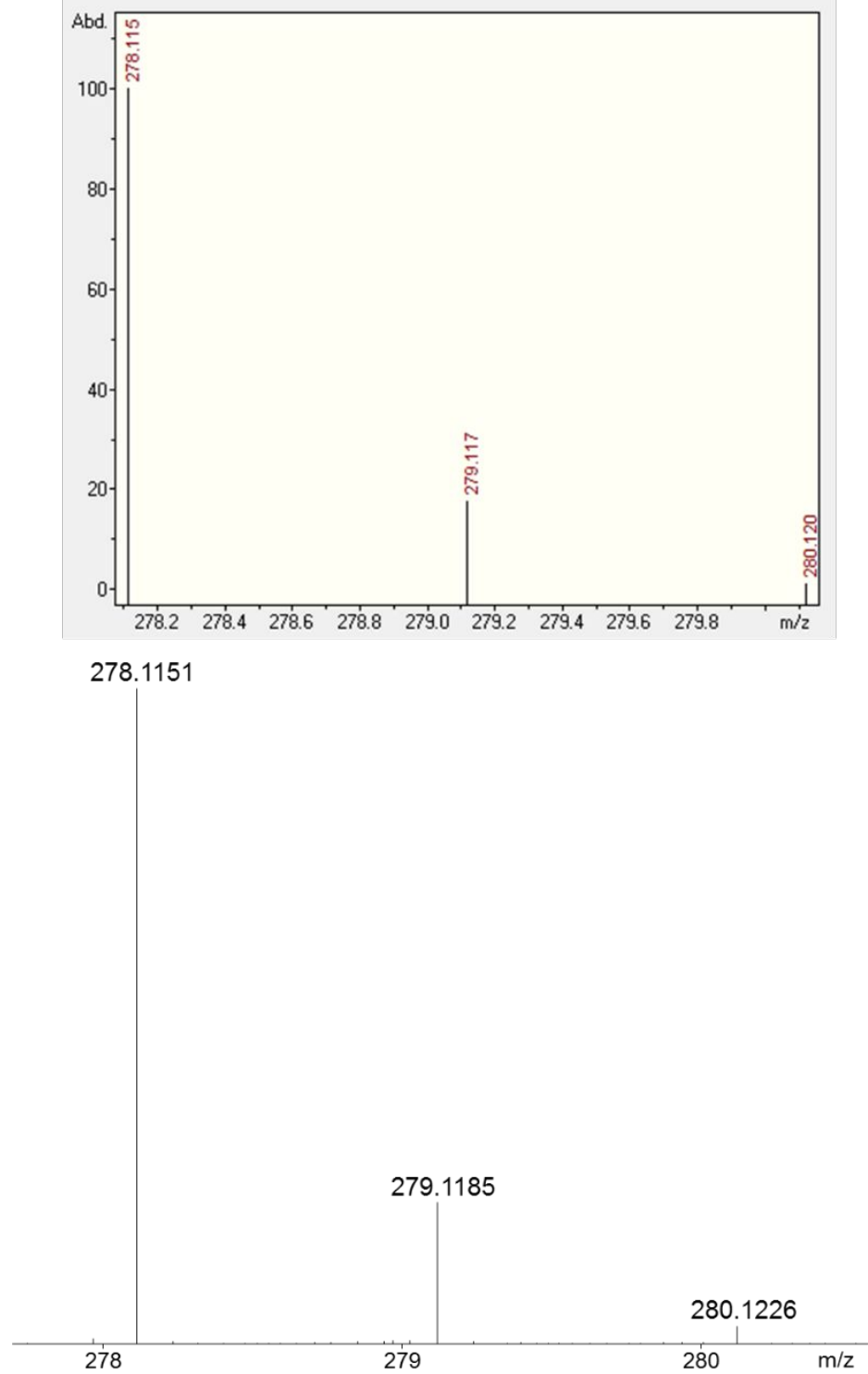

Figure S14: High-resolution ESI mass spectra of 1. Top: calculated spectrum. Bottom: experimental spectrum. 

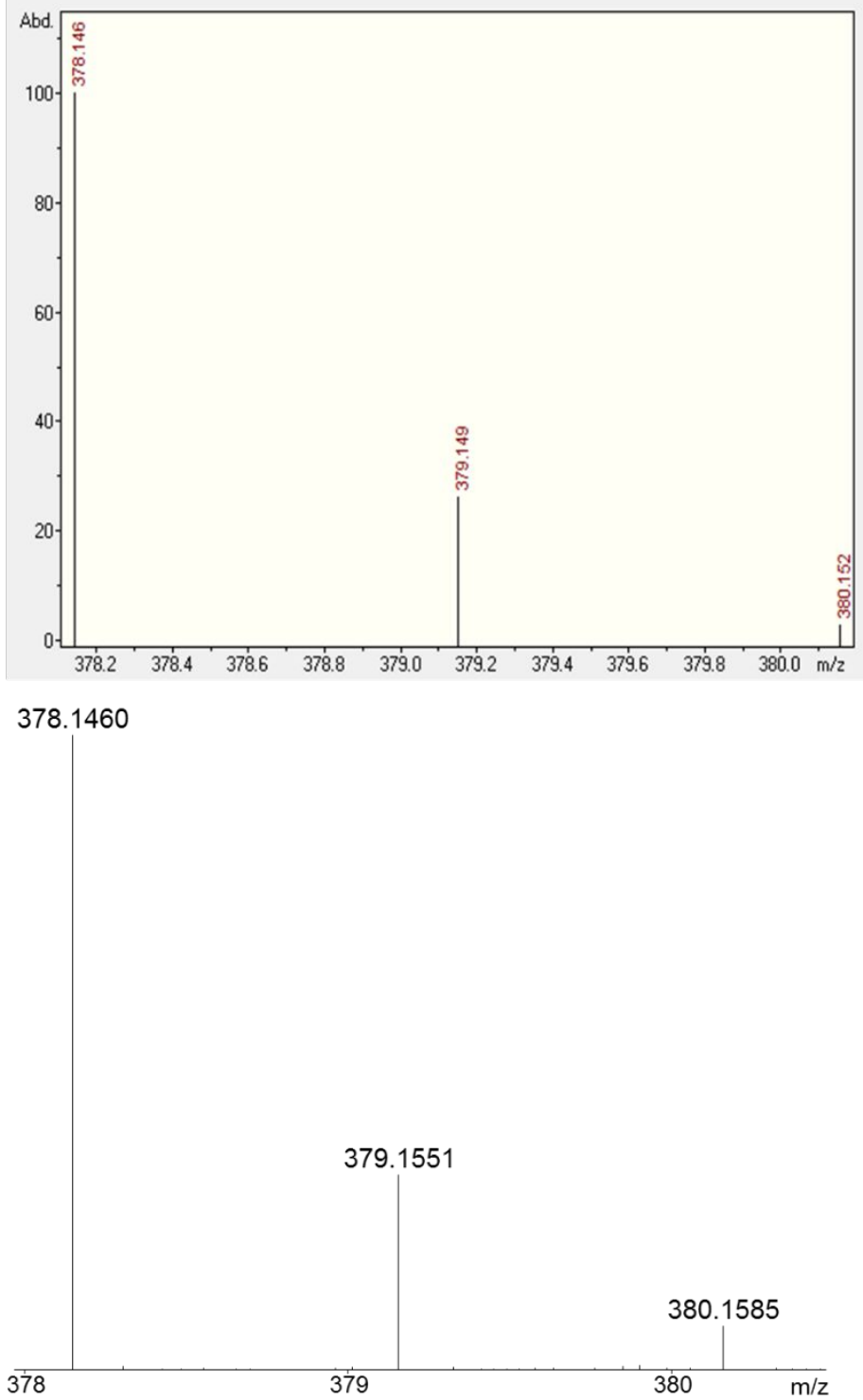

Figure S15: High-resolution ESI mass spectra of 2. Top: calculated spectrum. Bottom: experimental spectrum. 

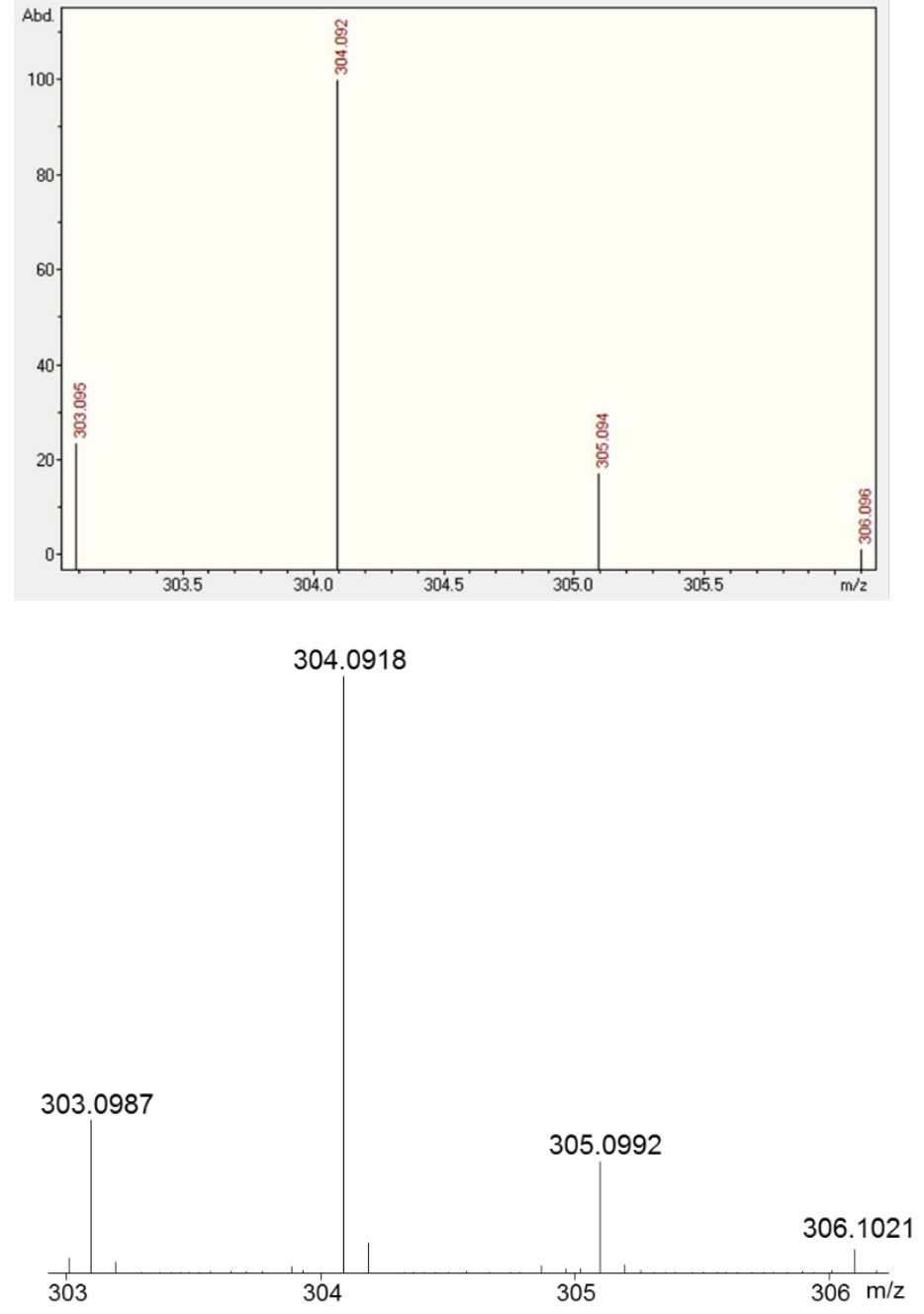

Figure S16: High-resolution ESI mass spectra of 1BF. Top: calculated spectrum. Bottom: experimental spectrum. 

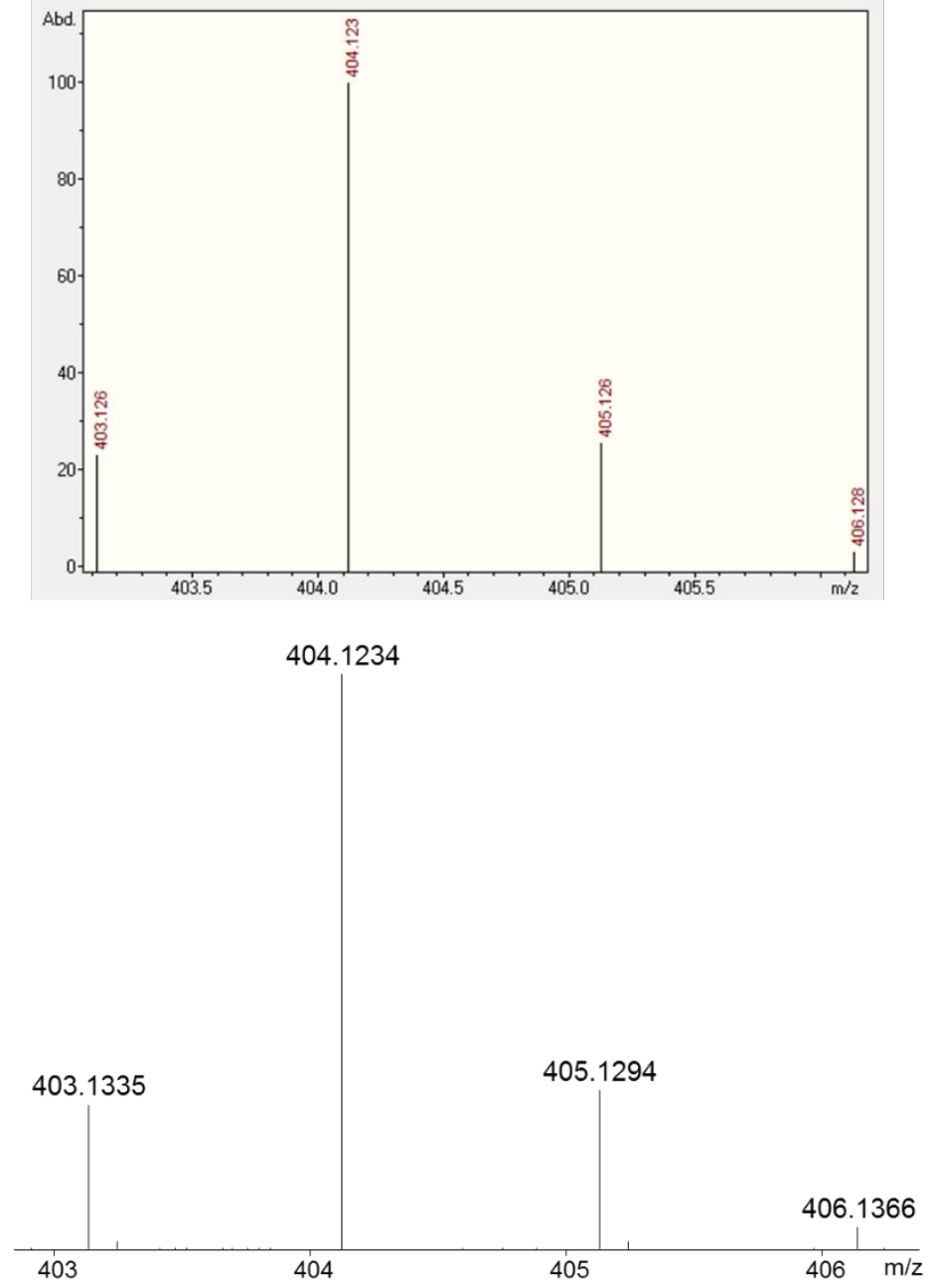

Figure S17: High-resolution ESI mass spectra of 2BF. Top: calculated spectrum. Bottom: experimental spectrum. 

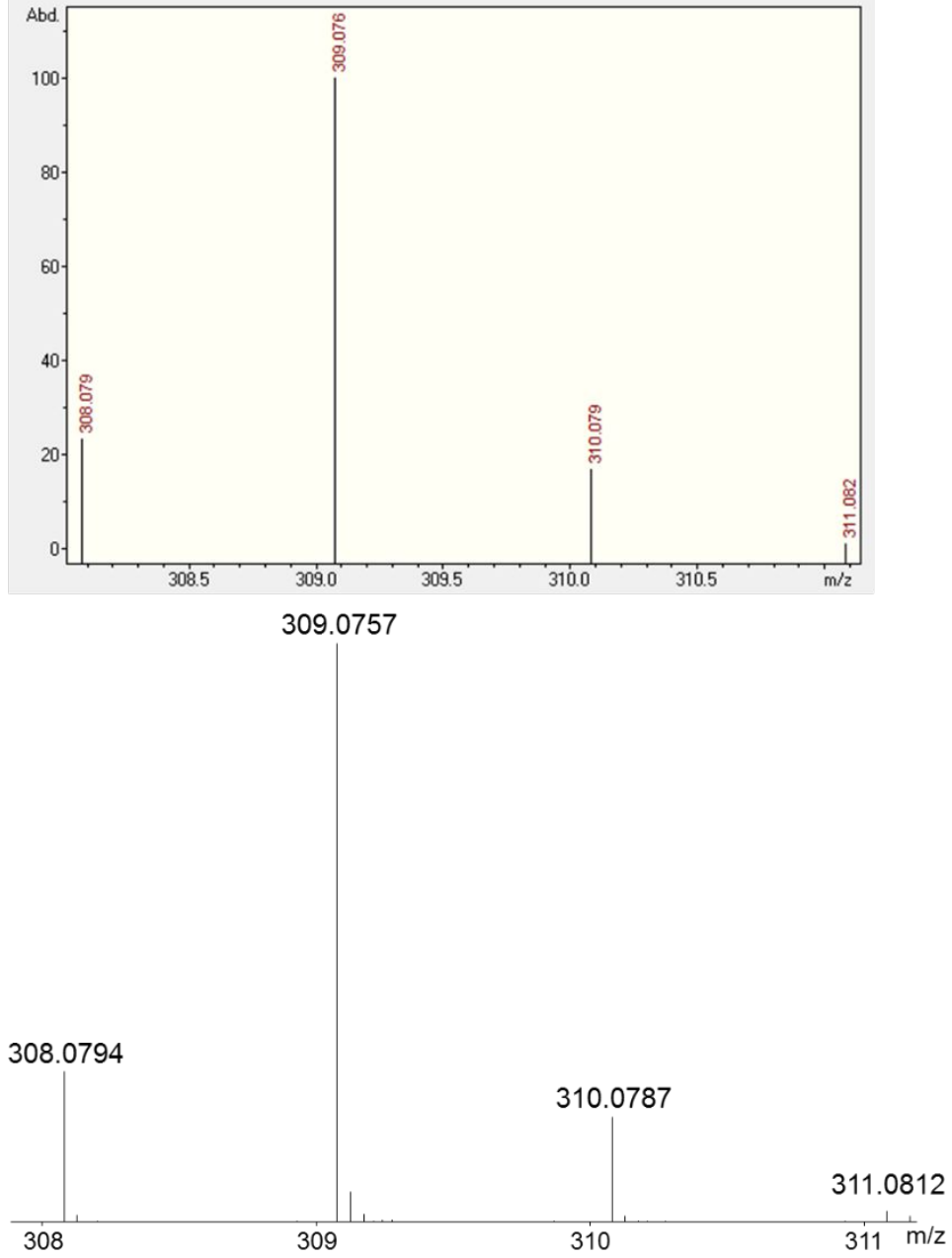

Figure S18: High-resolution ESI mass spectra of 3. Top: calculated spectrum. Bottom: experimental spectrum. 


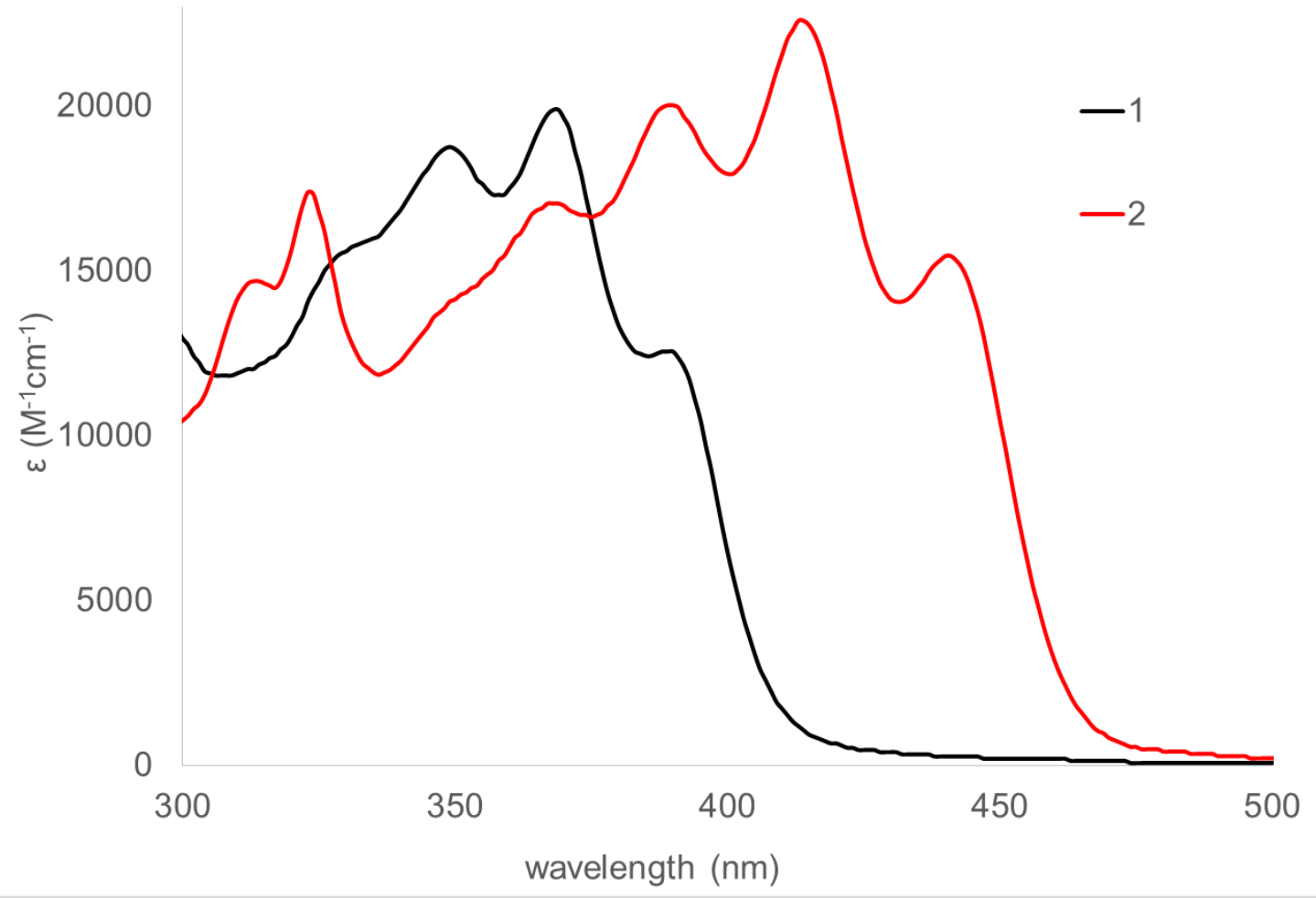

Figure S19: UV-visible spectra for compounds 1 and 2 in DMF.

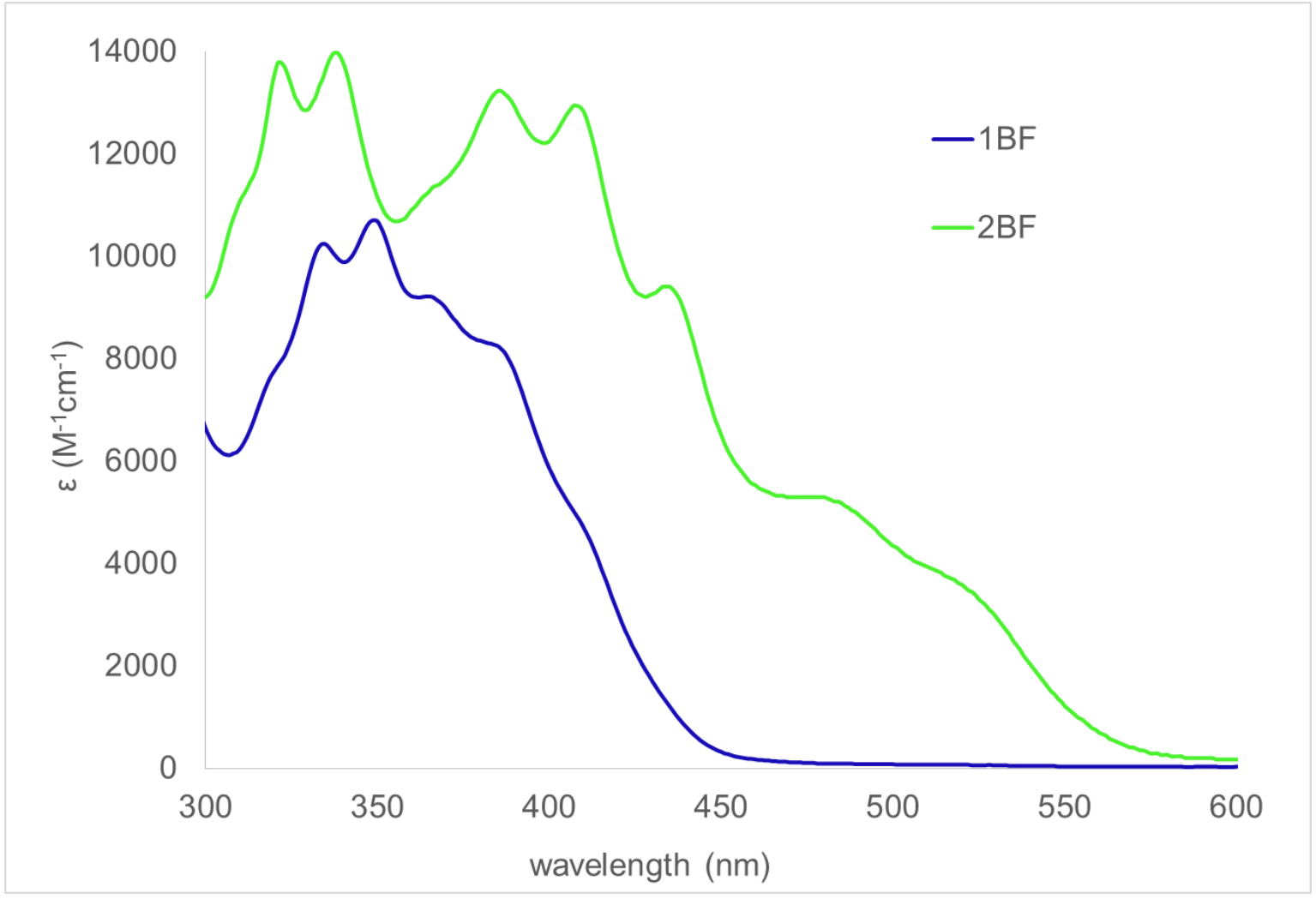

Figure S20: UV-visible spectra for compounds $\mathbf{1 B F}$ and $2 \mathbf{B F}$ in $\mathrm{CHCl}_{3}$. 


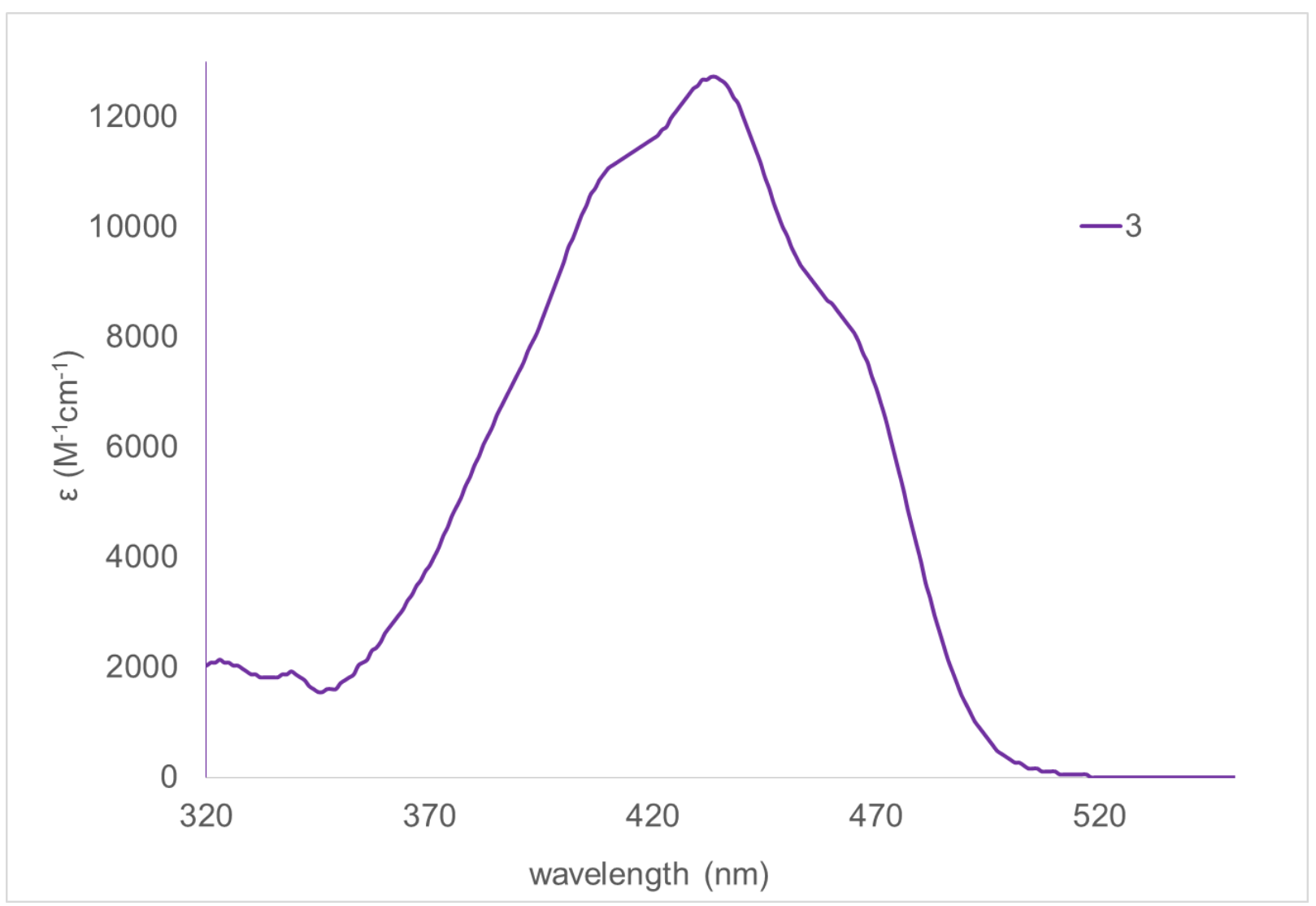

Figure S21: UV-visible spectra for compound 3 in DMF. 

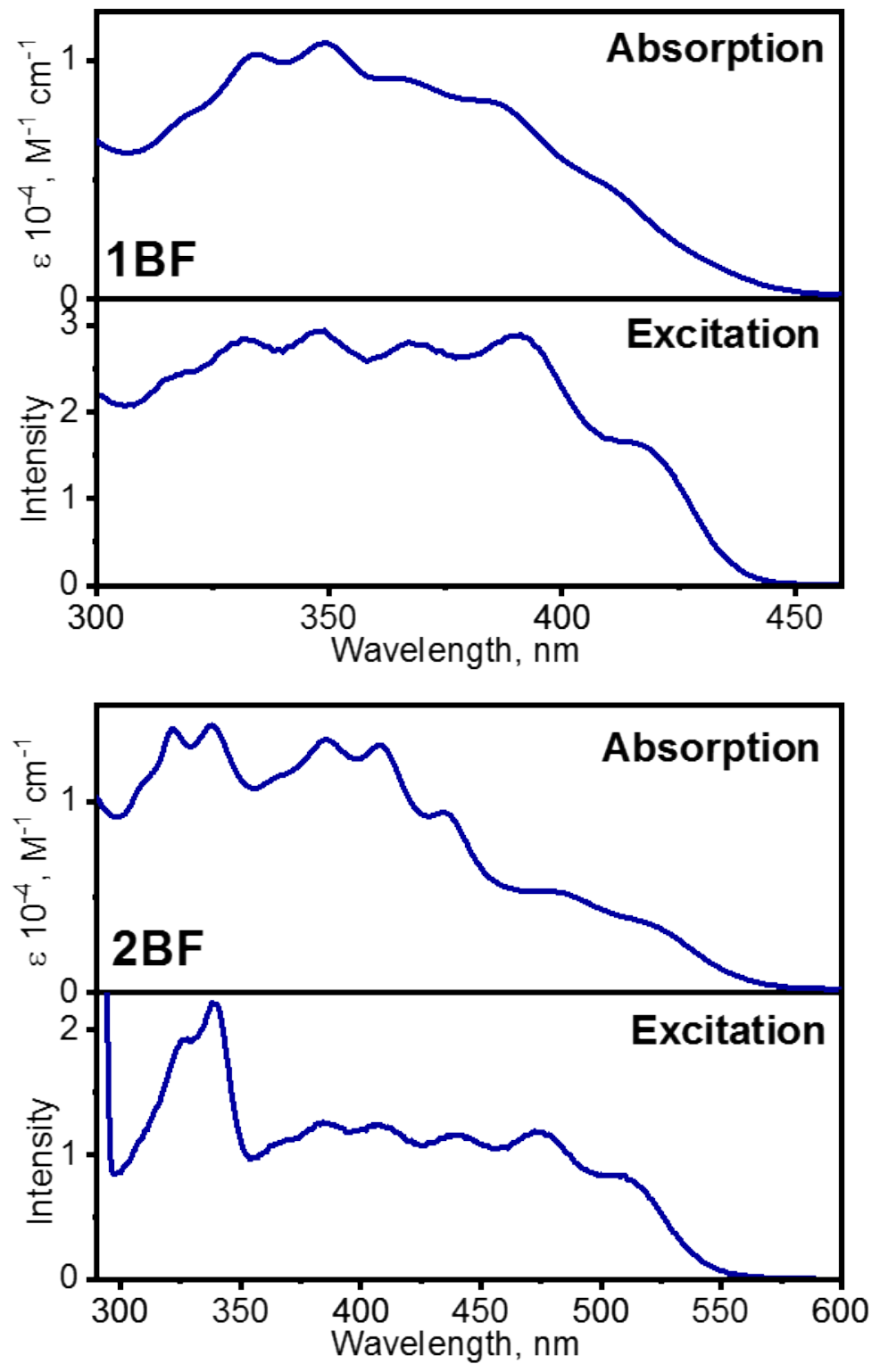

Figure S22: Absorption and excitation spectra for compounds $\mathbf{1 B F}$ and $\mathbf{2 B F}$ in $\mathrm{CHCl}_{3}$. 


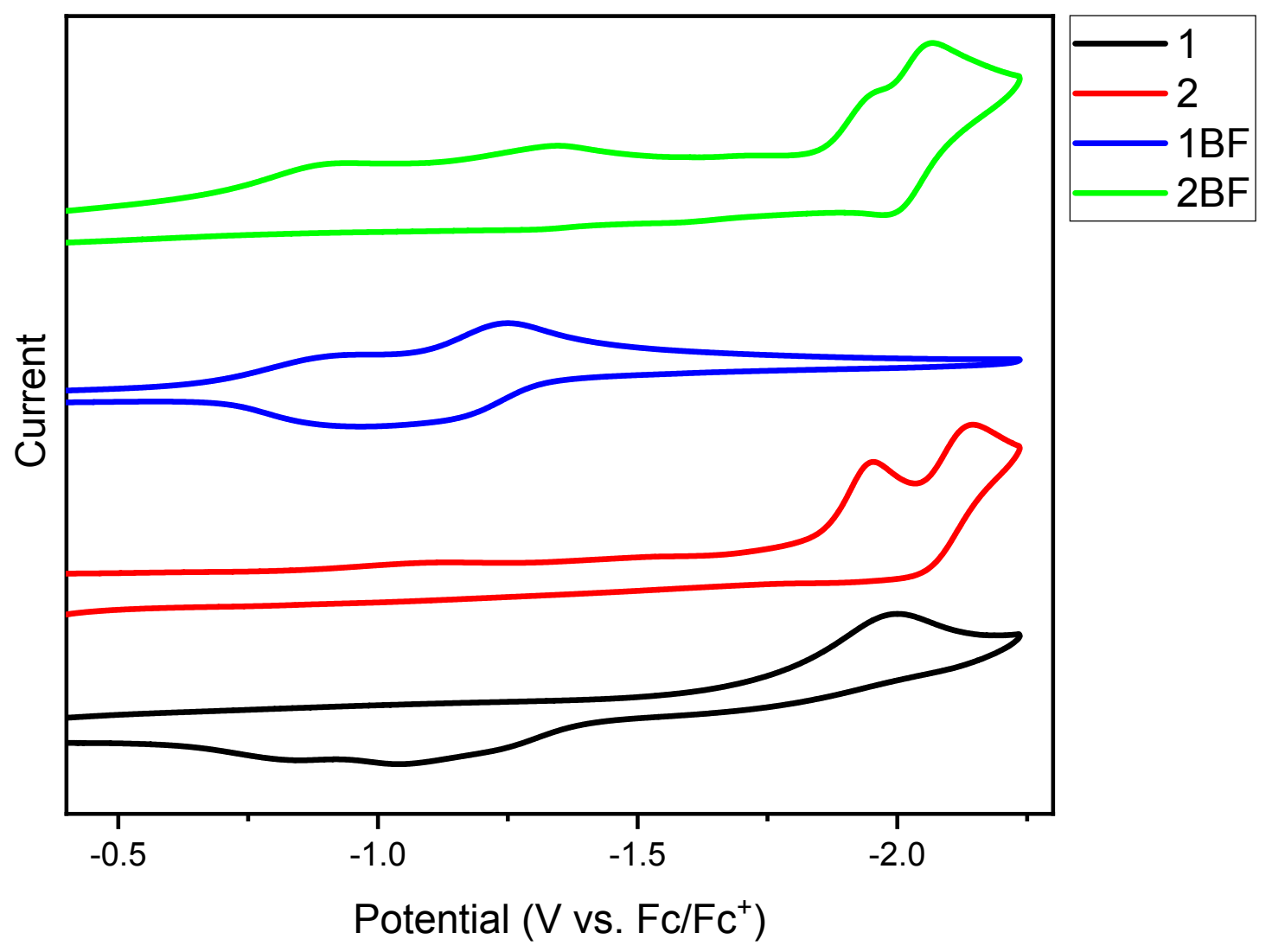

Figure S23: Cyclic voltammograms of 1, 2, 1BF, and 2BF in DMF/0.1 $\mathrm{TBAPF}_{6}$. 


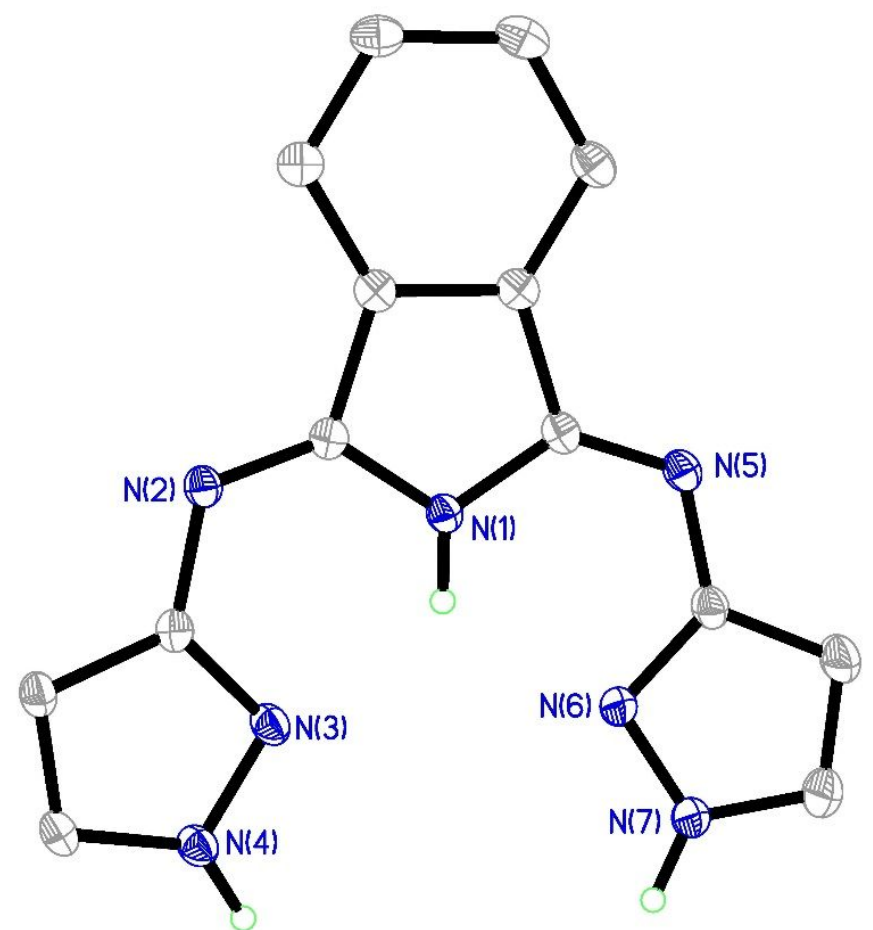

Figure S24: Structure of compound 1, with 35\% probability ellipsoids. Hydrogen atoms except on nitrogen atom positions have been omitted for clarity 


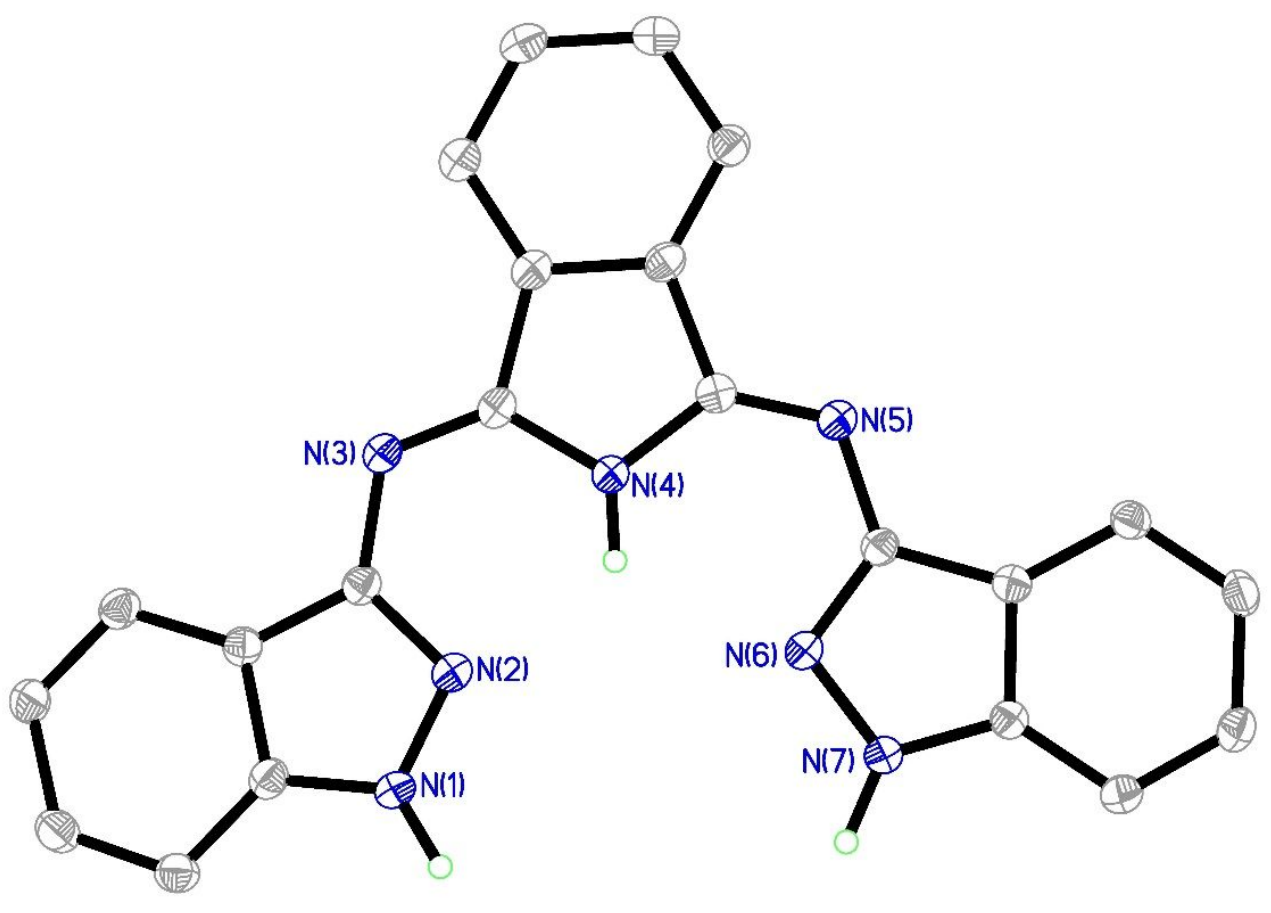

Figure S25: Structure of compound 2, with 35\% probability ellipsoids. Hydrogen atoms except on nitrogen atom positions have been omitted for clarity 


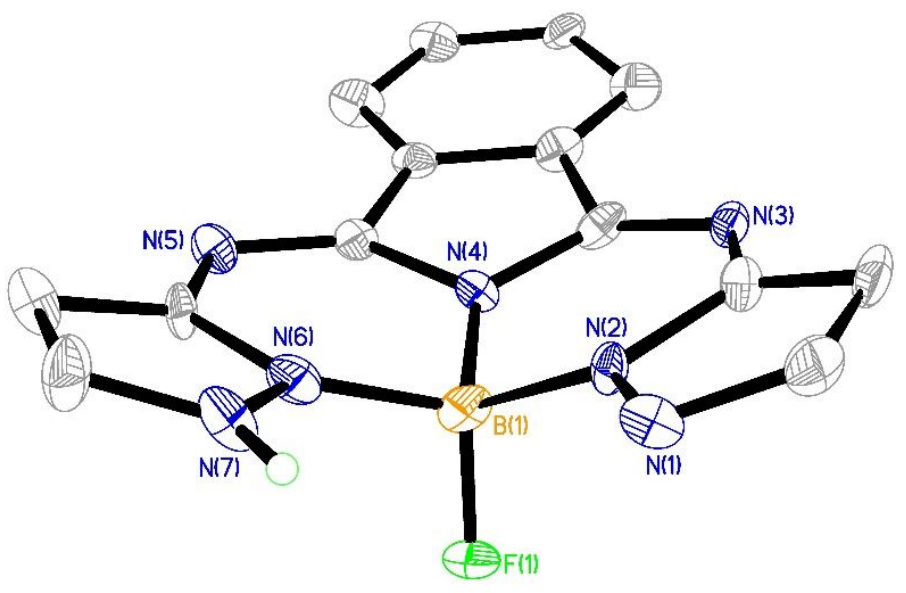

Figure S26: Structure of compound 1BF, with 35\% probability ellipsoids. Hydrogen atoms except on nitrogen atom positions have been omitted for clarity 


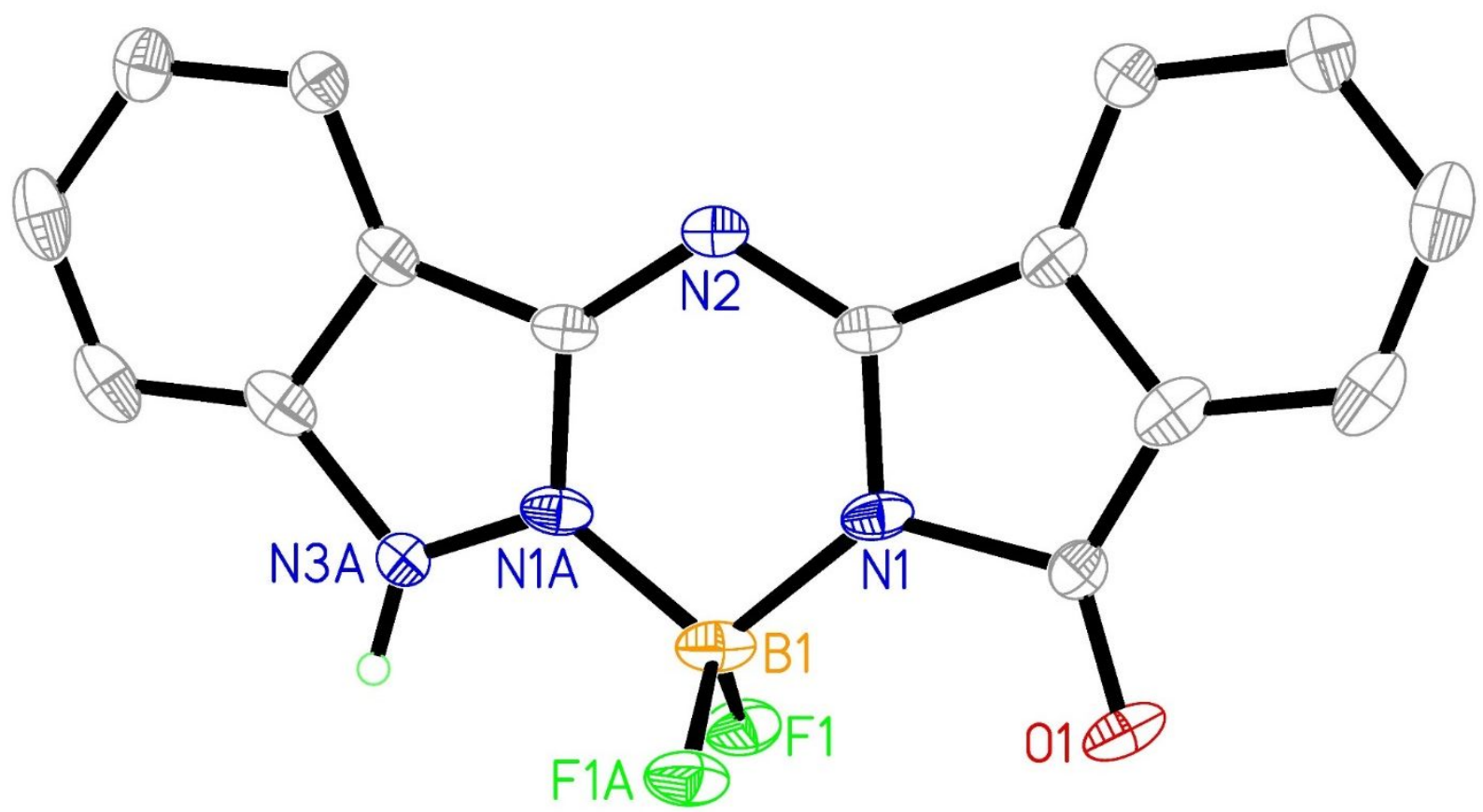

Figure S27: Structure of compound 3, with 35\% probability ellipsoids. Hydrogen atoms except on nitrogen atom positions have been omitted for clarity 


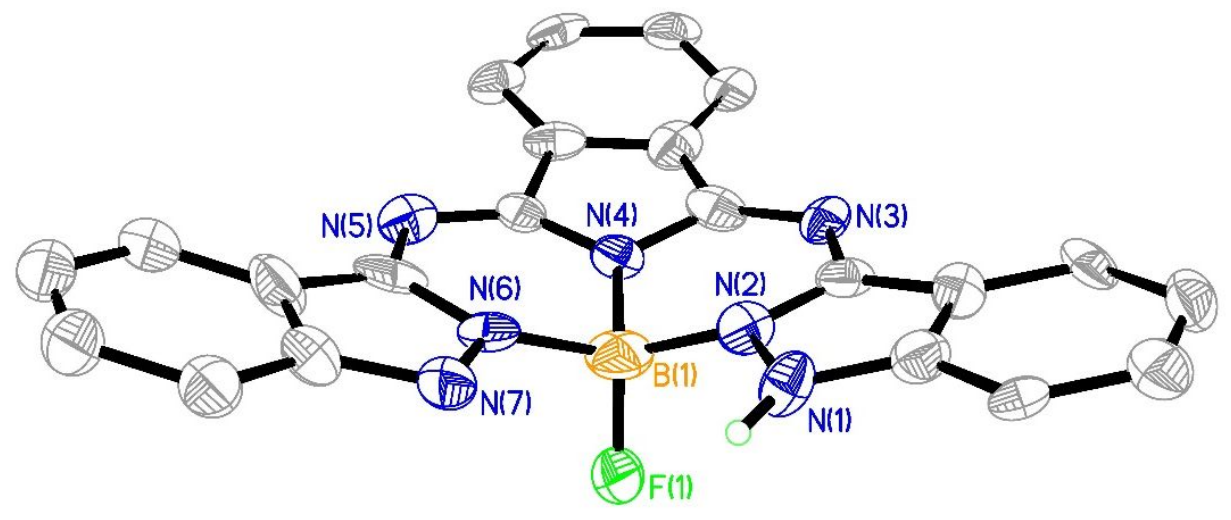

Figure S28: Structure of compound 2BF, with 35\% probability ellipsoids based on partial solution. Hydrogen atoms except on nitrogen atom positions have been omitted for clarity. $\mathrm{N}-\mathrm{N}$ hydrogen bond heteroatom distance $\sim 3.4 \AA$. 


\section{Compound}

$$
\text { HOMO }
$$

1

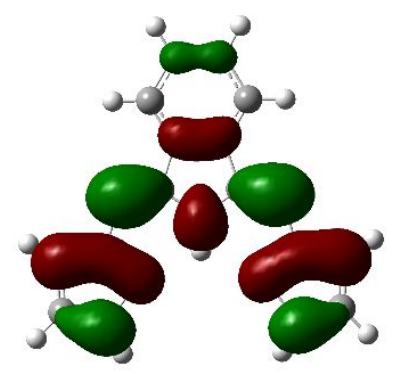

2

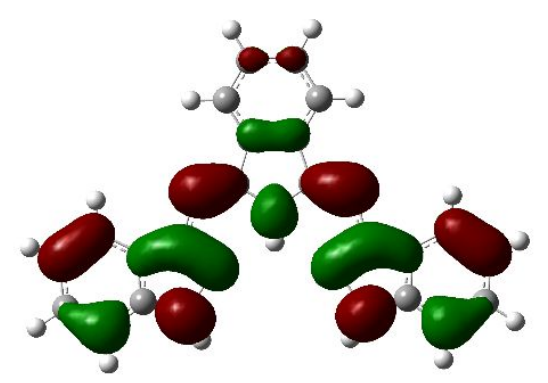

LUMO
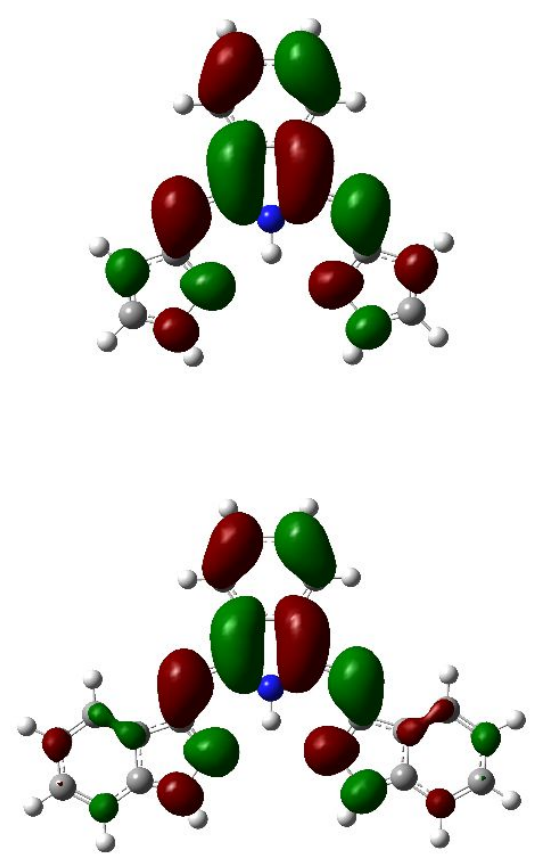

Figure S29: DFT-predicted frontier orbitals for compounds 1 and 2. 


\section{Compound}

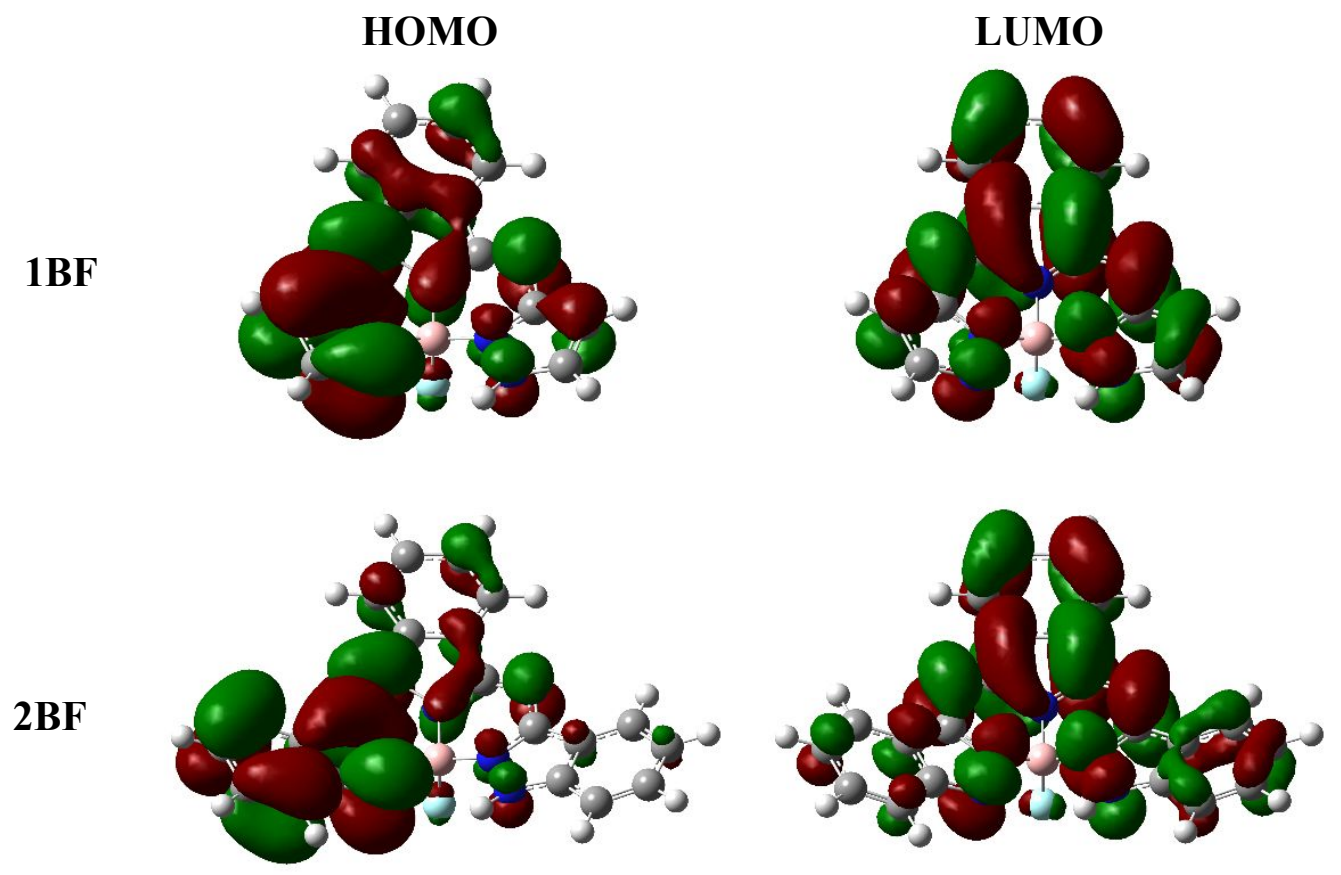

Figure S30: DFT-predicted frontier orbitals for compounds 1BF and 2BF. 

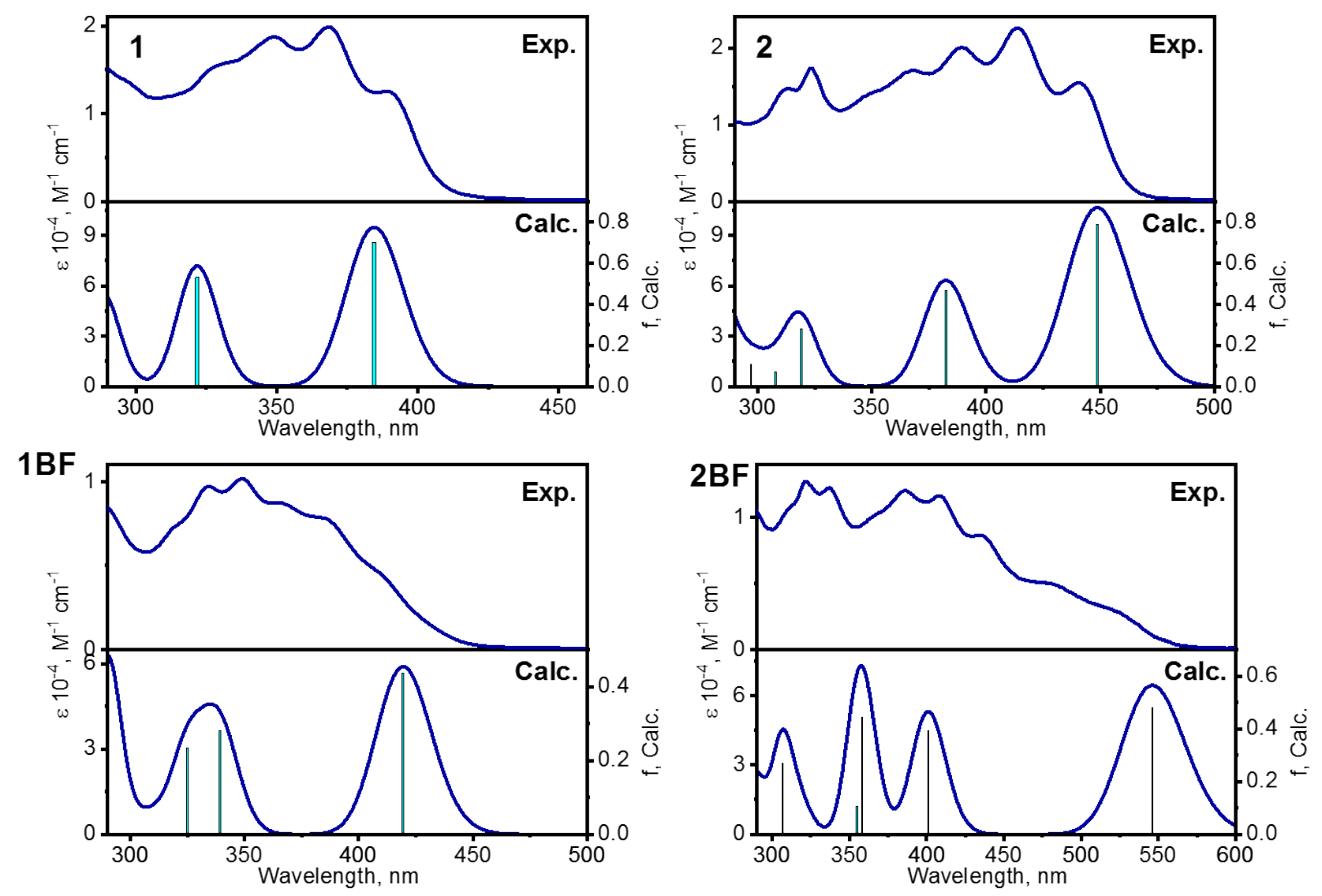

Figure S31: Experimental and B3LYP TDDFT-predicted spectra for compounds 1, 2, 1BF, and 2BF. 

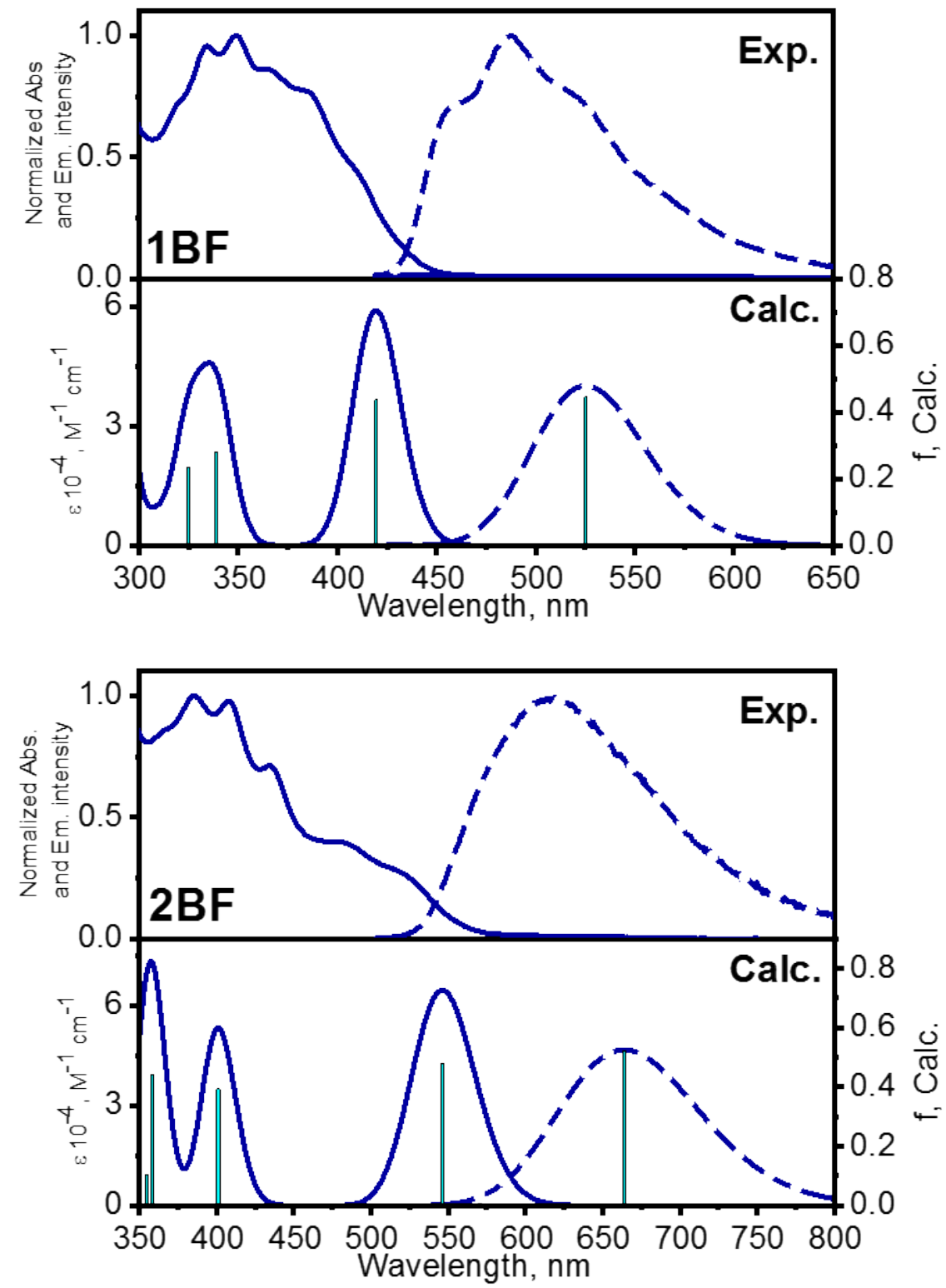

Figure S32: Experimental and B3LYP TDDFT-predicted excitation and emission spectra for compounds $1 \mathrm{BF}$ and $2 \mathrm{BF}$. 


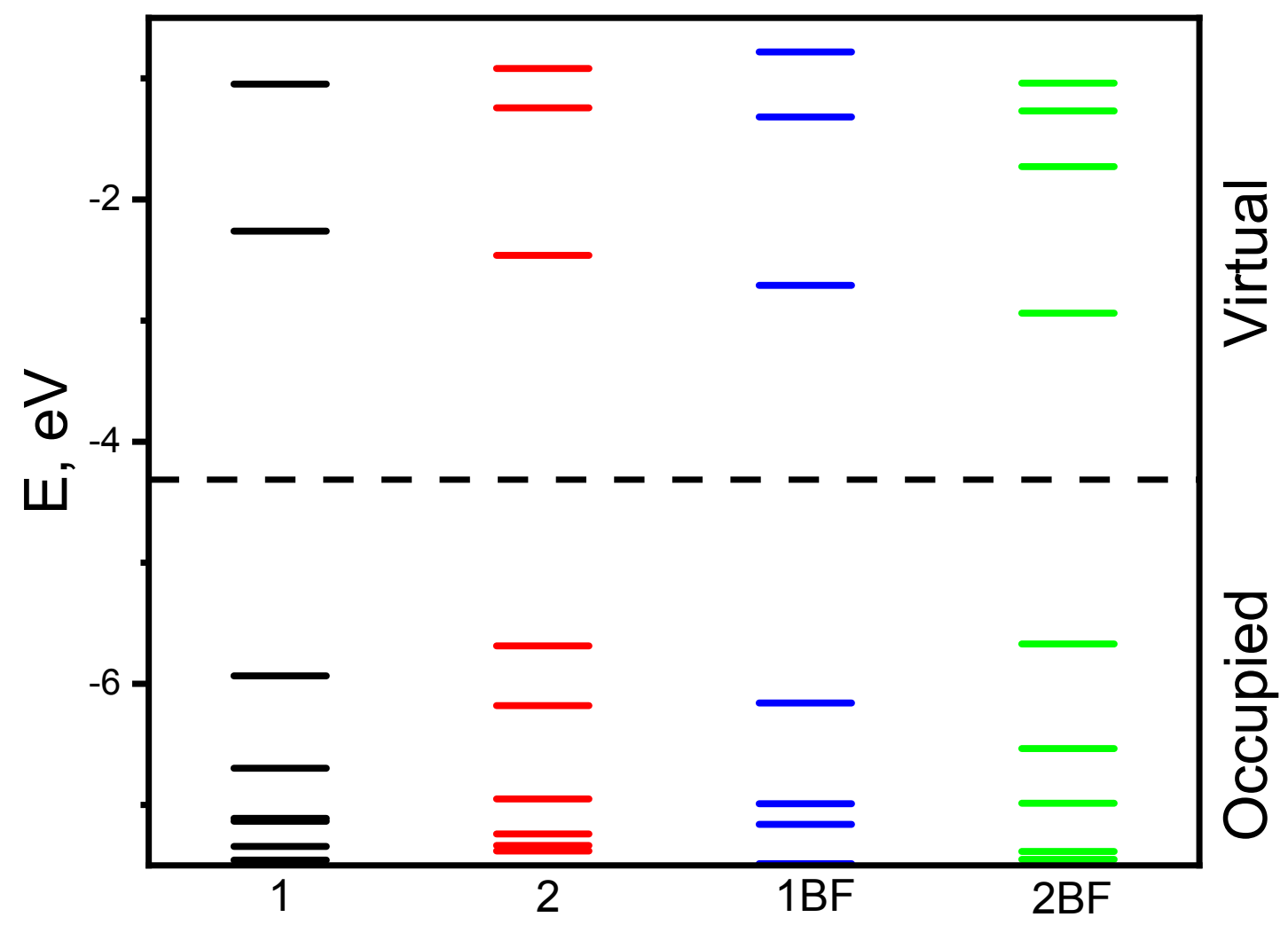

Fig S33: Relative energies of the frontier orbitals for compounds 1, 2, 1BF, and 2BF. 
Table S1: X-ray crystal data and structure parameters for compounds $\mathbf{1}$, and 2.

\begin{tabular}{|c|c|c|}
\hline Compound & 1 & 2 \\
\hline $\mathrm{CCDC}$ & 2052188 & 2052189 \\
\hline Empirical formula & $\mathrm{C}_{31} \mathrm{H}_{29} \mathrm{~N}_{15} \mathrm{O}$ & $\mathrm{C}_{22} \mathrm{H}_{15} \mathrm{~N}_{7}$ \\
\hline Formula weight & 627.69 & 377.41 \\
\hline Crystal system & Monoclinic & Monoclinic \\
\hline Space group & $\mathrm{P} 2_{1} / \mathrm{c}$ & $\mathrm{P} 21 / \mathrm{c}$ \\
\hline $\mathrm{a} / \AA$ & $10.2918(2)$ & $14.1029(7)$ \\
\hline $\mathrm{b} / \AA$ & $18.5703(3)$ & $23.6368(13)$ \\
\hline $\mathrm{c} / \AA$ & $15.8997(2)$ & $11.1420(6)$ \\
\hline$\alpha\left(^{\circ}\right)$ & 90 & 90 \\
\hline$\beta\left(^{\circ}\right)$ & $98.5810(10)$ & $93.727(3)$ \\
\hline$\gamma\left({ }^{\circ}\right)$ & 90 & 90 \\
\hline Volume $\left(\AA^{3}\right)$ & $3004.76(9)$ & $3706.3(3)$ \\
\hline $\mathrm{Z}$ & 4 & 8 \\
\hline $\operatorname{Dc}\left(\mathrm{Mg} / \mathrm{m}^{3}\right)$ & 1.388 & 1.353 \\
\hline$\mu\left(\mathrm{mm}^{-1}\right)$ & 0.759 & 0.690 \\
\hline $\mathrm{F}(000)$ & 1312 & 1568 \\
\hline reflns collected & 18150 & 23861 \\
\hline indep. reflns & 5250 & 6300 \\
\hline GOF on $\mathrm{F}^{2}$ & 1.033 & 1.011 \\
\hline $\mathrm{R} 1\left(\right.$ on $\left.\mathrm{F}_{\mathrm{o}}^{2}, \mathrm{I}>2 \sigma(\mathrm{I})\right)$ & 0.0543 & 0.0416 \\
\hline $\mathrm{wR} 2\left(\right.$ on $\left.\mathrm{F}_{\mathrm{o}}^{2}, \mathrm{I}>2 \sigma(\mathrm{I})\right)$ & 0.1483 & 0.0941 \\
\hline R1 (all data) & 0.0660 & 0.0657 \\
\hline wR2 (all data) & 0.1583 & 0.1059 \\
\hline
\end{tabular}


Table S2: X-ray crystal data and structure parameters for compounds 1BF and 3.

\begin{tabular}{ccc}
\hline Compound & 1BF & $\mathbf{3}$ \\
\hline CCDC & 2052190 & 2052191 \\
\hline Empirical formula & $\mathrm{C}_{20} \mathrm{H}_{24} \mathrm{BFN}_{8}$ & $\mathrm{C}_{15} \mathrm{H}_{9} \mathrm{BF}_{2} \mathrm{~N}_{4} \mathrm{O}$ \\
\hline Formula weight & 406.28 & 310.07 \\
\hline Crystal system & Monoclinic & Monoclinic \\
\hline Space group & $\mathrm{Pn}$ & $\mathrm{C} 2 / \mathrm{c}$ \\
\hline $\mathrm{a} / \AA$ & $9.327(3)$ & $14.7987(12)$ \\
\hline $\mathrm{b} / \AA$ & $22.100(5)$ & $14.1332(14)$ \\
\hline $\mathrm{c} / \AA$ & $24.878(6)$ & $6.9968(7)$ \\
\hline$\alpha\left(^{\circ}\right)$ & 90 & 60 \\
\hline$\beta\left({ }^{\circ}\right)$ & $92.46(2)$ & $116.300(3)$ \\
\hline$\gamma\left({ }^{\circ}\right)$ & 90 & 90 \\
\hline $\mathrm{Volume}\left(\AA^{3}\right)$ & $5123(3)$ & $1311.9(2)$ \\
\hline $\mathrm{Z}$ & 8 & 4 \\
\hline $\mathrm{Dc}\left(\mathrm{Mg} / \mathrm{m}^{3}\right)$ & 1.054 & 1.574 \\
\hline$\mu\left(\mathrm{mm}^{-1}\right)$ & 0.072 & 0.122 \\
\hline $\mathrm{F}(000)$ & 1712 & 632 \\
\hline reflns collected & 48943 & 11909 \\
\hline indep. reflns & 18565 & 1629 \\
\hline $\mathrm{GOF}$ on $\mathrm{F}^{2}$ & 1.072 & 1.225 \\
\hline $\mathrm{R} 1\left(\right.$ on $\left.\mathrm{F}_{\mathrm{o}}^{2}, \mathrm{I}>2 \sigma(\mathrm{I})\right)$ & 0.0709 & 0.0556 \\
\hline $\mathrm{wR} 2\left(\right.$ on $\left.\mathrm{F}_{\mathrm{o}}^{2}, \mathrm{I}>2 \sigma(\mathrm{I})\right)$ & 0.1819 & 0.1206 \\
\hline $\mathrm{R} 1($ all data) & 0.1073 & 0.0674 \\
\hline $\mathrm{wR} 2($ all data) & 0.1991 & 0.1238 \\
\hline
\end{tabular}


Table S3: Selected bond lengths for compounds 1BF and 3.

\begin{tabular}{|l|c|}
\hline & Compound 1BF \\
\hline $\mathrm{B}-\mathrm{N}_{(\text {pyrazole })}(\AA)$ & $1.503(8), 1.518(7), 1.495(7), 1.512(8)$, \\
& $1.489(7), 1.558(7), 1.487(8), 1.552(8)$ \\
\hline $\mathrm{B}-\mathrm{N}_{(\text {indoline })}(\AA)$ & $1.518(7), 1.519(8), 1.541(7), 1.501(8)$ \\
\hline $\mathrm{B}-\mathrm{F}(\AA)$ & $1.389(7), 1.436(7), 1.402(6), 1.434(7)$ \\
\hline $\mathrm{N}-\mathrm{N}$ hydrogen bond \\
heteroatom distance $(\AA)$ & $3.277(7), 3.224(7), 3.341(7), 3.273(8)$ \\
\hline & \multicolumn{1}{|c|}{ Compound 3 } \\
\hline $\mathrm{C}=\mathrm{O}(\AA)$ & $1.217(7)$ \\
\hline
\end{tabular}


Table S4: B3LYP TDDFT-predicted energies and expansion coefficients for compound $\mathbf{1}$ (only excited states with $f>0.05$ and $\lambda>270 \mathrm{~nm}$ are listed).

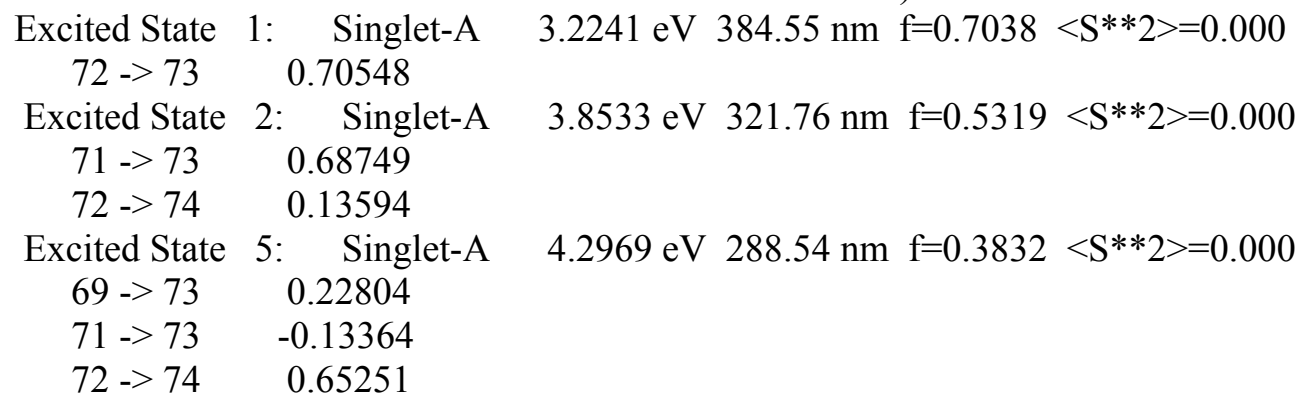

Table S5: B3LYP TDDFT-predicted energies and expansion coefficients for compound 2 (only excited states with $f>0.05$ and $\lambda>270 \mathrm{~nm}$ are listed).

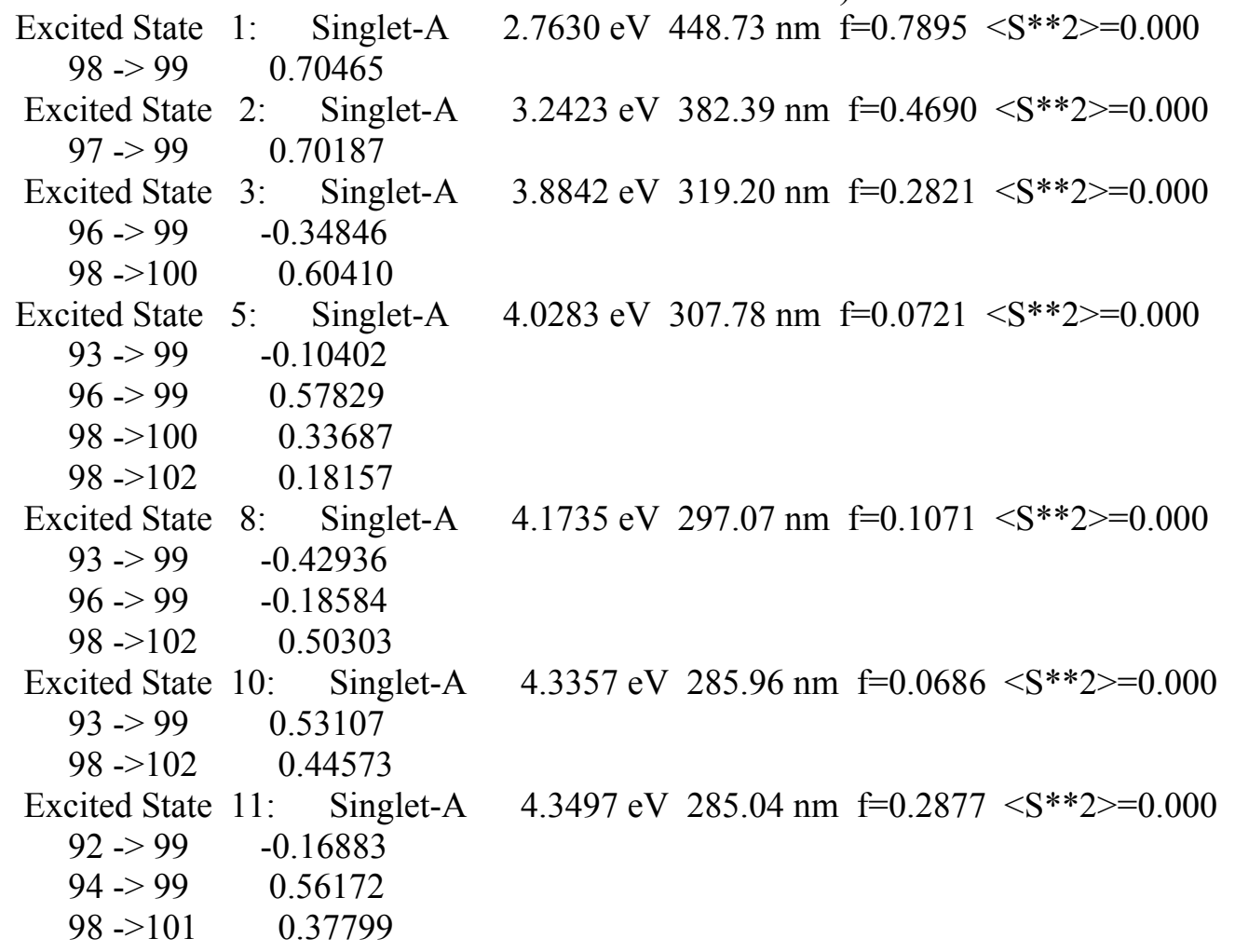


Table S6: B3LYP TDDFT-predicted energies and expansion coefficients for compound 1BF (only excited states with $\mathrm{f}>0.05$ and $\lambda>270 \mathrm{~nm}$ are listed).

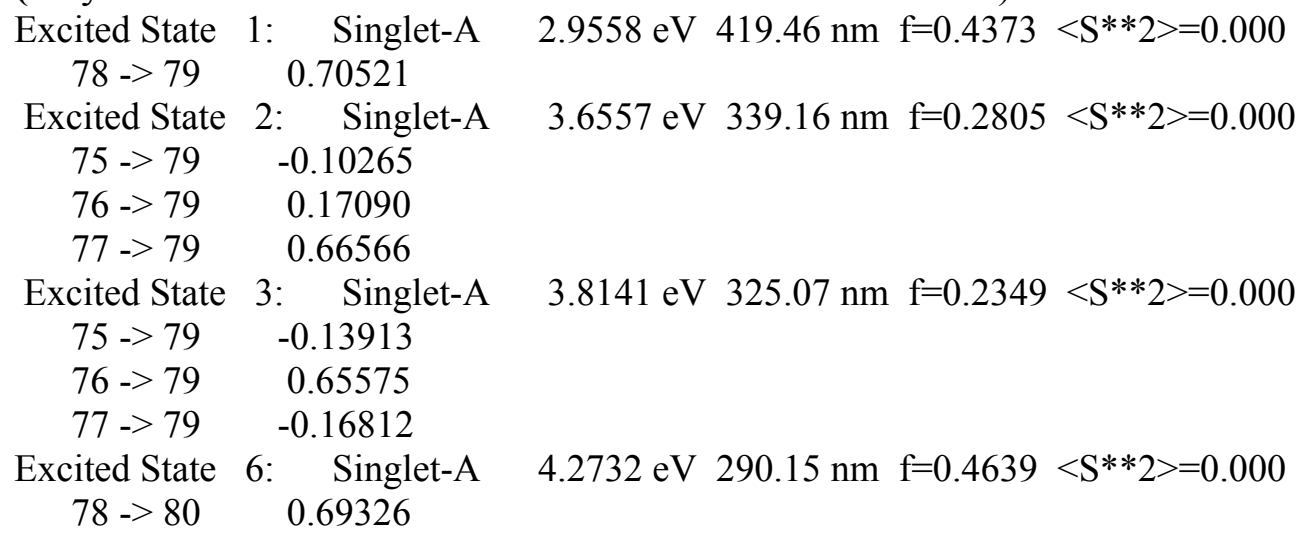

Table S7: B3LYP TDDFT-predicted energies and expansion coefficients for compound 2BF (only excited states with $\mathrm{f}>0.05$ and $\lambda>270 \mathrm{~nm}$ are listed).

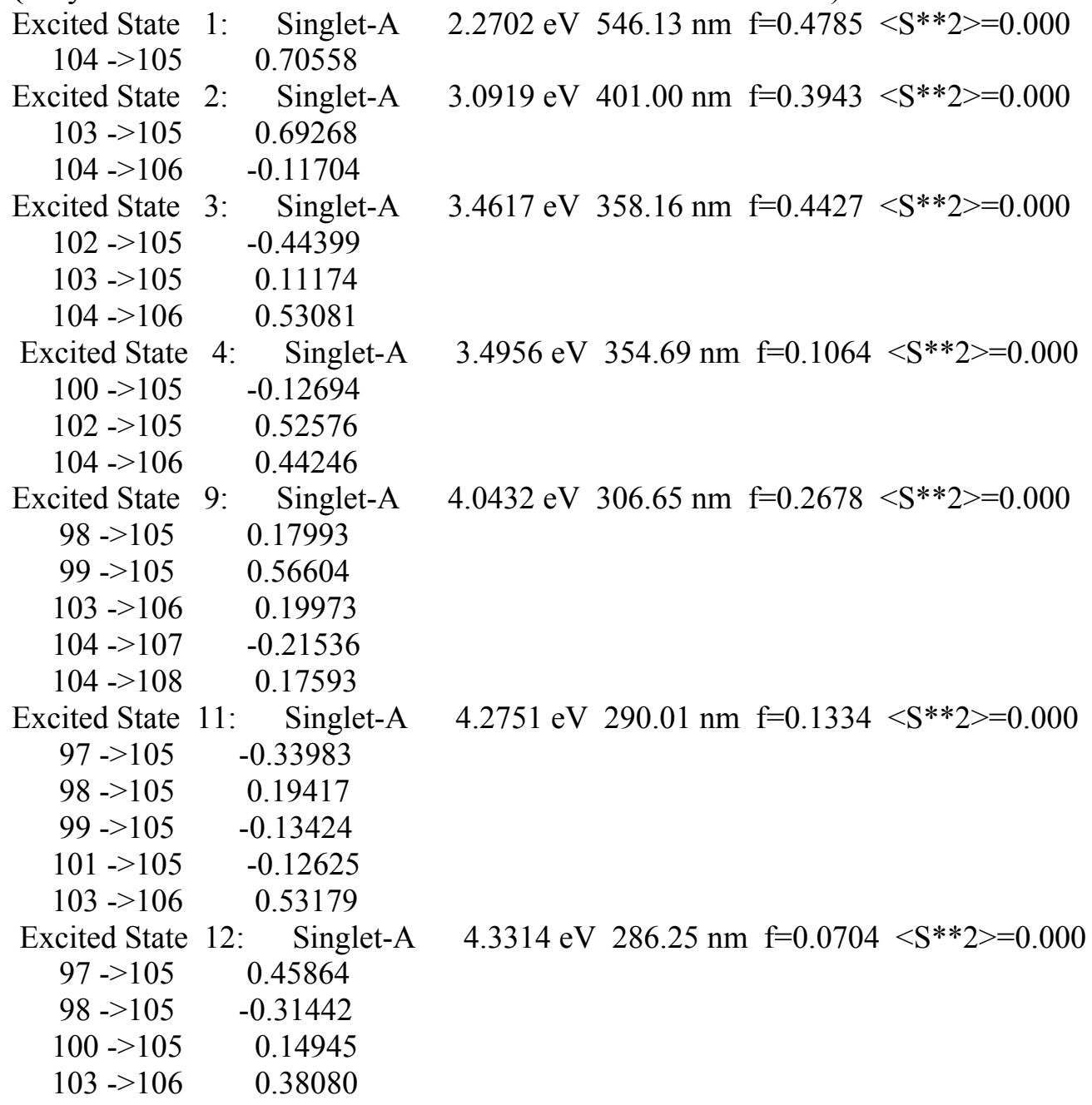


Table S8: The first three B3LYP TDDFT-predicted emission energies and expansion coefficients for compound $\mathbf{1 B F}$.

Excited State 1: $\quad$ Singlet-A $\quad 2.3605 \mathrm{eV} 525.25 \mathrm{~nm} \mathrm{f}=0.4461<\mathrm{S}^{* *} 2>=0.000$ $\begin{array}{ll}78->79 & 0.70649\end{array}$

Excited State 2: $\quad$ Singlet-A $\quad 3.3294 \mathrm{eV} \quad 372.39 \mathrm{~nm} \mathrm{f}=0.3107<\mathrm{S} * * 2>=0.000$ $74 \rightarrow 79 \quad 0.14009$

$76->79 \quad 0.21987$

$77->79 \quad 0.64369$

Excited State 3: $\quad$ Singlet-A $\quad 3.4650 \mathrm{eV} \quad 357.81 \mathrm{~nm} \mathrm{f}=0.1711<\mathrm{S} * * 2>=0.000$ $75 \rightarrow 79 \quad-0.18636$

$76->79 \quad 0.63339$

$77->79 \quad-0.21499$

Table S9: The first three B3LYP TDDFT-predicted emission energies and expansion coefficients for compound $\mathbf{2 B F}$.

Excited State 1: Singlet-A $\quad 1.8675 \mathrm{eV} \quad 663.91 \mathrm{~nm} \mathrm{f}=0.5188<\mathrm{S}^{* *} 2>=0.000$ $104->105 \quad 0.70698$

Excited State 2: Singlet-A $2.8556 \mathrm{eV} \quad 434.17 \mathrm{~nm} \mathrm{f}=0.3730<\mathrm{S} * * 2>=0.000$ $103->105 \quad 0.69223$ $104->106 \quad-0.12552$

Excited State 3: $\quad$ Singlet-A $\quad 3.2016 \mathrm{eV} \quad 387.26 \mathrm{~nm} \mathrm{f}=0.1492<\mathrm{S} * * 2>=0.000$ $100->105 \quad-0.10344$ $102 \rightarrow 105 \quad 0.65818$ $104->106 \quad-0.20652$ 
Table S10: B3LYP DFT ground state optimized geometry of compound 1.

\begin{tabular}{|c|c|c|c|c|}
\hline \multirow{3}{*}{$\begin{array}{l}\text { Center } \\
\text { Number } \\
1\end{array}$} & \multirow{2}{*}{$\begin{array}{l}\text { Atomic } \\
\text { Number }\end{array}$} & \multirow{2}{*}{$\begin{array}{c}\text { Atomic } \\
\text { Type }\end{array}$} & \multicolumn{2}{|c|}{ Coordinates (Angstror } \\
\hline & & & $\mathrm{X} \quad \mathrm{Y}$ & $Y \quad Z$ \\
\hline & 6 & 1.193232 & 0.673781 & -0.000226 \\
\hline 2 & 6 & 0.810434 & 2.097530 & -0.000115 \\
\hline 3 & 6 & 1.590588 & 3.247855 & -0.000010 \\
\hline 4 & 1 & 2.672939 & 3.185477 & -0.000041 \\
\hline 5 & 6 & 0.935791 & 4.480110 & 0.000157 \\
\hline 6 & 1 & 1.517014 & 5.395930 & 0.000226 \\
\hline 7 & 6 & -0.463887 & 4.553232 & 0.000214 \\
\hline 8 & 1 & -0.946500 & 5.524633 & 0.000329 \\
\hline 9 & 6 & -1.243602 & 3.395977 & 0.000086 \\
\hline 10 & 1 & -2.326482 & 3.447006 & 0.000151 \\
\hline 11 & 6 & -0.587752 & 2.170502 & -0.000071 \\
\hline 12 & 6 & -1.116737 & 0.794197 & -0.000182 \\
\hline 13 & 6 & 2.715905 & -1.108513 & -0.000088 \\
\hline 14 & 6 & 4.041502 & -1.618201 & 0.000199 \\
\hline 15 & 1 & 4.956623 & -1.047821 & 0.000330 \\
\hline 16 & 6 & 3.896633 & -2.987457 & 0.000264 \\
\hline 17 & 1 & 4.616208 & -3.791100 & 0.000458 \\
\hline 18 & 6 & -2.816606 & -0.820227 & $7-0.000051$ \\
\hline 19 & 6 & -4.188071 & -1.189075 & 50.000308 \\
\hline 20 & 1 & -5.038765 & -0.526440 & 0.000582 \\
\hline 21 & 6 & -4.186606 & -2.565981 & 0.000239 \\
\hline 22 & 1 & -4.986042 & -3.290229 & 0.000418 \\
\hline 23 & 1 & -0.054605 & -1.051323 & $3-0.000338$ \\
\hline 24 & 7 & -0.001987 & -0.038334 & -0.000362 \\
\hline 25 & 7 & 2.404646 & 0.241718 & -0.000160 \\
\hline 26 & 7 & 1.819732 & -2.111620 & -0.000050 \\
\hline 27 & 7 & 2.563208 & -3.232294 & 0.000093 \\
\hline 28 & 1 & 2.105635 & -4.130092 & 0.000130 \\
\hline 29 & 7 & -2.366523 & 0.490275 & -0.000070 \\
\hline 30 & 7 & -2.029810 & -1.911247 & -0.000245 \\
\hline 31 & 7 & -2.885943 & -2.948423 & -0.000114 \\
\hline 32 & 1 & -2.524217 & -3.888946 & -0.000232 \\
\hline
\end{tabular}

$\mathrm{E}_{h}=-923.570437$ Hartree 
Table S11: B3LYP DFT ground state optimized geometry of compound 2.

\begin{tabular}{|c|c|c|c|c|}
\hline \multirow{2}{*}{$\begin{array}{l}\text { Center } \\
\text { Number }\end{array}$} & Atomic & \multirow{2}{*}{$\begin{array}{c}\text { Atomic } \\
\text { Type }\end{array}$} & \multicolumn{2}{|c|}{ Coordinates (Angstroms } \\
\hline & Number & & $\begin{array}{ll}X & Y\end{array}$ & Y $\quad \mathrm{Z}$ \\
\hline 1 & 7 & -2.387406 & 0.964921 & -0.000052 \\
\hline 2 & 7 & 0.000012 & 0.566441 & -0.000370 \\
\hline 3 & 7 & 2.714486 & -2.515851 & -0.001067 \\
\hline 4 & 7 & 2.387423 & 0.964944 & -0.000571 \\
\hline 5 & 7 & -1.935820 & -1.408968 & -0.000746 \\
\hline 6 & 7 & -2.714496 & -2.515858 & -0.000815 \\
\hline 7 & 7 & 1.935814 & -1.408952 & -0.001135 \\
\hline 8 & 6 & 4.129602 & -0.808234 & 0.000063 \\
\hline 9 & 6 & 4.048179 & -2.218992 & -0.000204 \\
\hline 10 & 6 & 2.763384 & -0.362311 & -0.000553 \\
\hline 11 & 6 & 0.700499 & 2.740731 & -0.000299 \\
\hline 12 & 6 & -2.763386 & -0.362322 & -0.000161 \\
\hline 13 & 6 & -4.129599 & -0.808227 & 0.000217 \\
\hline 14 & 6 & -1.155164 & 1.340125 & -0.000201 \\
\hline 15 & 6 & 1.155172 & 1.340129 & -0.000459 \\
\hline 16 & 6 & 5.383704 & -0.177751 & 0.000841 \\
\hline 17 & 1 & 5.456258 & 0.904244 & 0.001025 \\
\hline 18 & 6 & -4.048185 & -2.218981 & -0.000183 \\
\hline 19 & 6 & -0.700512 & 2.740725 & -0.000089 \\
\hline 20 & 6 & 5.192808 & -3.031221 & 0.000300 \\
\hline 21 & 1 & 5.122785 & -4.113047 & 0.000114 \\
\hline 22 & 6 & 1.419415 & 3.930455 & -0.000249 \\
\hline 23 & 1 & 2.503429 & 3.925639 & -0.000398 \\
\hline 24 & 6 & 6.416830 & -2.385028 & 0.001089 \\
\hline 25 & 1 & 7.326579 & -2.976301 & 0.001522 \\
\hline 26 & 6 & -5.192812 & -3.031210 & 0.000066 \\
\hline 27 & 1 & -5.122778 & -4.113035 & -0.000227 \\
\hline 28 & 6 & 6.516167 & -0.973243 & 0.001338 \\
\hline 29 & 1 & 7.498903 & -0.514194 & 0.001938 \\
\hline 30 & 6 & -5.383703 & -0.177745 & 0.000856 \\
\hline 31 & 1 & -5.456263 & 0.904251 & 0.001134 \\
\hline 32 & 6 & 0.700834 & 5.126470 & 0.000010 \\
\hline 33 & 1 & 1.233264 & 6.071418 & 0.000054 \\
\hline 34 & 6 & -6.416837 & -2.385022 & 0.000710 \\
\hline 35 & 1 & -7.326589 & -2.976290 & 0.000923 \\
\hline 36 & 6 & 0 & -0.973 & 0.001100 \\
\hline 37 & 1 & -7.498900 & -0.514180 & 0.001595 \\
\hline 38 & 6 & -1.419414 & 3.930461 & 0.000186 \\
\hline 39 & 1 & -2.503431 & 3.925687 & 0.000339 \\
\hline 40 & 6 & -0.700823 & 5.126470 & 0.000227 \\
\hline 41 & 1 & -1.233262 & 6.071414 & 0.000425 \\
\hline 42 & 1 & 0.000000 & -0.447828 & -0.000854 \\
\hline 43 & 1 & -2.277859 & -3.423392 & -0.001020 \\
\hline 44 & 1 & 2.277830 & -3.423380 & -0.000898 \\
\hline
\end{tabular}

$\mathrm{E}_{h}=-1230.921115$ Hartree 
Table S12: B3LYP DFT ground state optimized geometry of compound 1BF.

\begin{tabular}{|c|c|c|c|c|c|}
\hline \multirow{2}{*}{$\begin{array}{l}\text { Center } \\
\text { Number }\end{array}$} & \multicolumn{2}{|l|}{ Atomic } & \multirow{2}{*}{$\begin{array}{l}\text { Atomic } \\
\text { Type }\end{array}$} & \multicolumn{2}{|c|}{ Coordinates (Angstroms) } \\
\hline & Number & & & $\mathrm{X} \quad \mathrm{Y}$ & $\mathrm{Z}$ \\
\hline 1 & 5 & 0 & 1.243446 & 0.052406 & 0.639158 \\
\hline 2 & 6 & 0 & 3.014608 & -2.975128 & -0.570013 \\
\hline 3 & 1 & 0 & 3.941432 & -3.461008 & -0.828701 \\
\hline 4 & 6 & 0 & 1.719585 & -3.454946 & -0.513346 \\
\hline 5 & 1 & 0 & 1.381980 & -4.455986 & -0.722918 \\
\hline 6 & 6 & 0 & 0.911983 & -2.357510 & -0.152278 \\
\hline 7 & 6 & 0 & -0.971588 & -1.125882 & 0.148683 \\
\hline 8 & 6 & 0 & -2.381008 & -0.710629 & 0.050328 \\
\hline 9 & 6 & 0 & -3.550404 & -1.448254 & -0.076285 \\
\hline 10 & 1 & 0 & -3.529859 & -2.532023 & -0.077111 \\
\hline 11 & 6 & 0 & -4.753158 & -0.748880 & -0.197702 \\
\hline 12 & 1 & 0 & -5.684492 & -1.297239 & -0.285009 \\
\hline 13 & 6 & 0 & -4.774501 & 0.650724 & -0.214777 \\
\hline 14 & 1 & 0 & -5.722893 & 1.166970 & -0.314893 \\
\hline 15 & 6 & 0 & -3.595614 & 1.392951 & -0.110817 \\
\hline 16 & 1 & 0 & -3.612495 & 2.476442 & -0.138085 \\
\hline 17 & 6 & 0 & -2.401456 & 0.699465 & 0.032898 \\
\hline 18 & 6 & 0 & -1.005397 & 1.158954 & 0.118884 \\
\hline 19 & 6 & 0 & 0.873270 & 2.416033 & -0.173946 \\
\hline 20 & 6 & 0 & 1.674978 & 3.495814 & -0.534494 \\
\hline 21 & 1 & 0 & 1.344049 & 4.490165 & -0.790285 \\
\hline 22 & 6 & 0 & 2.984200 & 2.986088 & -0.513330 \\
\hline 23 & 1 & 0 & 3.907935 & 3.498053 & -0.745199 \\
\hline 24 & 9 & 0 & 1.526206 & -0.005196 & 2.022671 \\
\hline 25 & 7 & 0 & 2.993955 & -1.670172 & -0.239638 \\
\hline 26 & 1 & 0 & 3.739500 & -0.986680 & -0.232780 \\
\hline 27 & 7 & 0 & 1.726355 & -1.275053 & -0.001032 \\
\hline 28 & 7 & 0 & -0.455963 & -2.309922 & -0.022714 \\
\hline 29 & 7 & 0 & -0.234918 & 0.017372 & 0.318456 \\
\hline 30 & 7 & 0 & -0.507928 & 2.331839 & -0.087202 \\
\hline 31 & 7 & 0 & 1.722075 & 1.352728 & 0.039737 \\
\hline 32 & 7 & 0 & 3.014823 & 1.697956 & -0.154417 \\
\hline
\end{tabular}


Table S13: B3LYP DFT ground state optimized geometry of compound 2BF.

\begin{tabular}{|c|c|c|c|c|}
\hline \multirow{2}{*}{$\begin{array}{l}\text { Center } \\
\text { Number }\end{array}$} & Atomic & \multirow{2}{*}{$\begin{array}{r}\text { Atomic } \\
\text { Type }\end{array}$} & \multicolumn{2}{|c|}{ Coordinates (Angstrom } \\
\hline & Numbe & & X $\quad \mathrm{Y}$ & $\mathrm{Z}$ \\
\hline 1 & 9 & 0.008870 & -0.495834 & 2.399138 \\
\hline 2 & 7 & -1.271980 & -0.924444 & 0.413986 \\
\hline 3 & 7 & 0.010104 & 1.067392 & 0.513111 \\
\hline 4 & 7 & 1.646663 & -2.225330 & 0.364606 \\
\hline 5 & 7 & 2.321631 & 1.285670 & 0.085967 \\
\hline 6 & 7 & 1.348623 & -0.913790 & 0.445242 \\
\hline 7 & 7 & -2.312475 & 1.228439 & 0.134988 \\
\hline 8 & 7 & -1.628193 & -2.236319 & 0.331782 \\
\hline 9 & 1 & -0.890452 & -2.928504 & 0.291503 \\
\hline 10 & 6 & 0.690739 & 3.188865 & -0.002318 \\
\hline 11 & 6 & 3.480090 & -0.939617 & -0.174871 \\
\hline 12 & 6 & 4.818064 & -0.748625 & -0.577895 \\
\hline 13 & 1 & 5.225207 & 0.250181 & -0.694415 \\
\hline 14 & 6 & 1.380548 & 4.363537 & -0.274169 \\
\hline 15 & 1 & 2.464070 & 4.382664 & -0.295655 \\
\hline 16 & 6 & 3.753214 & -3.391355 & -0.278816 \\
\hline 17 & 1 & 3.349481 & -4.391951 & -0.166922 \\
\hline 18 & 6 & 2.399527 & -0.086662 & 0.137483 \\
\hline 19 & 6 & -0.721656 & 3.165298 & 0.007068 \\
\hline 20 & 6 & -1.131798 & 1.776869 & 0.257842 \\
\hline 21 & 6 & -2.340541 & -0.137441 & 0.151246 \\
\hline 22 & 6 & 1.150803 & 1.815477 & 0.239657 \\
\hline 23 & 6 & 2.943470 & -2.258096 & -0.025762 \\
\hline 24 & 6 & -3.732173 & -3.437786 & -0.344091 \\
\hline 25 & 1 & -3.340193 & -4.445524 & -0.279153 \\
\hline 26 & 6 & -0.765365 & 5.495019 & -0.504963 \\
\hline 27 & 1 & -1.315 & 6.408675 & -0.699645 \\
\hline 28 & 6 & -1.461744 & 4.311164 & -0.255088 \\
\hline 29 & 1 & -2.545366 & 4.286579 & -0.261494 \\
\hline 30 & 6 & 0.634827 & 5.519538 & -0.514295 \\
\hline 31 & 1 & 1.148650 & 6.452912 & -0.716095 \\
\hline 32 & 6 & -2.931696 & -2.320165 & -0.064405 \\
\hline 33 & 6 & -5.043893 & -3.188647 & -0.701389 \\
\hline 34 & 1 & -5.695978 & -4.026458 & -0.922738 \\
\hline 35 & 6 & -5.566127 & -1.874667 & -0.787242 \\
\hline 36 & 1 & -6.6 & -1.7 & -1.070269 \\
\hline 37 & 6 & 5.056515 & -3.177980 & -0.665604 \\
\hline 38 & 1 & 5.701093 & -4.028200 & -0.864034 \\
\hline 39 & 6 & -4.775048 & -0.775409 & -0.520487 \\
\hline 40 & 1 & -5.164207 & 0.233648 & -0.588303 \\
\hline 41 & 6 & -3.436811 & -0.999717 & -0.155316 \\
\hline 42 & 6 & 5.589167 & -1.865375 & -0.815476 \\
\hline 43 & 1 & 6.623232 & -1.751469 & -1.123302 \\
\hline 44 & 5 & 0.044508 & -0.363744 & 0.996767 \\
\hline
\end{tabular}

$\mathrm{E}_{h}=-1354.589433$ Hartree 
Table S14: B3LYP DFT excited state optimized geometry of compound 1BF.

\begin{tabular}{|c|c|c|c|c|c|}
\hline \multirow{2}{*}{$\begin{array}{l}\text { Center } \\
\text { Number }\end{array}$} & Atomic & & \multirow{2}{*}{$\begin{array}{l}\text { Atomic } \\
\text { Type }\end{array}$} & \multicolumn{2}{|c|}{ Coordinates (Angstroms } \\
\hline & Numbe & & & $\mathrm{X} \quad \mathrm{Y}$ & $\mathrm{Z}$ \\
\hline 1 & 5 & 0 & 1.262681 & 0.049077 & 0.526252 \\
\hline 2 & 6 & 0 & 3.074604 & -3.009195 & -0.417666 \\
\hline 3 & 1 & 0 & 4.015087 & -3.515226 & -0.567063 \\
\hline 4 & 6 & 0 & 1.786282 & -3.494702 & -0.363065 \\
\hline 5 & 1 & 0 & 1.470572 & -4.522223 & -0.435302 \\
\hline 6 & 6 & 0 & 0.940792 & -2.370719 & -0.182001 \\
\hline 7 & 6 & 0 & -0.977077 & -1.171766 & 0.139060 \\
\hline 8 & 6 & 0 & -2.357432 & -0.777948 & 0.047194 \\
\hline 9 & 6 & 0 & -3.545042 & -1.501953 & -0.090049 \\
\hline 10 & 1 & 0 & -3.537333 & -2.586240 & -0.100538 \\
\hline 11 & 6 & 0 & -4.740401 & -0.796878 & -0.205852 \\
\hline 12 & 1 & 0 & -5.671919 & -1.344443 & -0.302953 \\
\hline 13 & 6 & 0 & -4.769916 & 0.612237 & -0.203716 \\
\hline 14 & 1 & 0 & -5.719248 & 1.126695 & -0.300728 \\
\hline 15 & 6 & 0 & -3.592819 & 1.346747 & -0.084962 \\
\hline 16 & 1 & 0 & -3.607018 & 2.431121 & -0.095022 \\
\hline 17 & 6 & 0 & -2.390750 & 0.652206 & 0.048056 \\
\hline 18 & 6 & 0 & -1.022339 & 1.110325 & 0.156411 \\
\hline 19 & 6 & 0 & 0.781100 & 2.463035 & -0.114052 \\
\hline 20 & 6 & 0 & 1.580193 & 3.612955 & -0.400670 \\
\hline 21 & 1 & 0 & 1.218293 & 4.620217 & -0.531809 \\
\hline 22 & 6 & 0 & 2.873924 & 3.132989 & -0.492698 \\
\hline 23 & 1 & 0 & 3.783498 & 3.672042 & -0.713803 \\
\hline 24 & 9 & 0 & 1.631165 & -0.010730 & 1.888558 \\
\hline 25 & 7 & 0 & 3.037067 & -1.670418 & -0.257353 \\
\hline 26 & 1 & 0 & 3.776331 & -0.994817 & -0.387721 \\
\hline 27 & 7 & 0 & 1.744016 & -1.244834 & -0.170756 \\
\hline 28 & 7 & 0 & -0.402468 & -2.348250 & -0.047225 \\
\hline 29 & 7 & 0 & -0.234685 & 0.010624 & 0.294345 \\
\hline 30 & 7 & 0 & -0.547611 & 2.365431 & 0.050817 \\
\hline 31 & 7 & 0 & 1.672460 & 1.394797 & -0.061671 \\
\hline 32 & 7 & 0 & 2.935669 & 1.795516 & -0.266626 \\
\hline
\end{tabular}

$\mathrm{E}_{h}=-1047.234212$ Hartree 
Table S15: B3LYP DFT excited state optimized geometry of compound 2BF.

\begin{tabular}{|c|c|c|c|c|}
\hline \multirow{2}{*}{$\begin{array}{l}\text { Center } \\
\text { Number }\end{array}$} & Atomic & \multirow{2}{*}{$\begin{array}{r}\text { Atomic } \\
\text { Type }\end{array}$} & \multicolumn{2}{|c|}{ Coordinates (Angstrom } \\
\hline & Number & & $\begin{array}{ll}X & Y\end{array}$ & Z \\
\hline 1 & 9 & -0.089671 & -0.652375 & 2.198389 \\
\hline 2 & 7 & -1.294602 & -0.932275 & 0.132276 \\
\hline 3 & 7 & 0.045633 & 1.024952 & 0.410585 \\
\hline 4 & 7 & 1.641097 & -2.281131 & 0.154629 \\
\hline 5 & 7 & 2.408459 & 1.212404 & 0.197007 \\
\hline 6 & 7 & 1.342589 & -0.967839 & 0.296752 \\
\hline 7 & 7 & -2.285386 & 1.264594 & -0.000096 \\
\hline 8 & 7 & -1.767963 & -2.232541 & 0.205962 \\
\hline 9 & 1 & -1.119522 & -2.959815 & -0.065973 \\
\hline 10 & 6 & 0.793066 & 3.132712 & 0.038755 \\
\hline 11 & 6 & 3.538007 & -1.011951 & -0.112052 \\
\hline 12 & 6 & 4.893210 & -0.822348 & -0.361018 \\
\hline 13 & 1 & 5.327872 & 0.170872 & -0.374707 \\
\hline 14 & 6 & 1.540587 & 4.292258 & -0.153487 \\
\hline 15 & 1 & 2.624294 & 4.263922 & -0.125265 \\
\hline 16 & 6 & 3.772727 & -3.452636 & -0.342417 \\
\hline 17 & 1 & 3.343373 & -4.447899 & -0.335516 \\
\hline 18 & 6 & 2.434264 & -0.137546 & 0.149587 \\
\hline 19 & 6 & -0.631866 & 3.157298 & -0.013853 \\
\hline 20 & 6 & -1.088844 & 1.800351 & 0.165917 \\
\hline 21 & 6 & -2.362176 & -0.077730 & -0.012531 \\
\hline 22 & 6 & 1.191060 & 1.751689 & 0.252053 \\
\hline 23 & 6 & 2.967961 & -2.319914 & -0.100549 \\
\hline 24 & 6 & -4.004366 & -3.295324 & -0.229678 \\
\hline 25 & 1 & -3.671598 & -4.324551 & -0.165969 \\
\hline 26 & 6 & -0.546795 & 5.513227 & -0.433794 \\
\hline 27 & 1 & -1.051438 & 6.455969 & -0.616447 \\
\hline 28 & 6 & -1.303340 & 4.356326 & -0.261427 \\
\hline 29 & 1 & -2.385971 & 4.387205 & -0.313455 \\
\hline 30 & 6 & 0.858869 & 5.486318 & -0.380791 \\
\hline 31 & 1 & 1.416026 & 6.405342 & -0.523876 \\
\hline 32 & 6 & -3.111660 & -2.228345 & -0.074730 \\
\hline 33 & 6 & -5.333920 & -2.975125 & -0.463253 \\
\hline 34 & 1 & -6.054362 & -3.776510 & -0.586658 \\
\hline 35 & 6 & -5.776472 & -1.637282 & -0.541823 \\
\hline 36 & 1 & -6.826726 & -1.436796 & -0.721253 \\
\hline 37 & 6 & 5.125605 & -3.246396 & -0.584359 \\
\hline 38 & 1 & 5.768313 & -4.099729 & -0.769648 \\
\hline 39 & 6 & -4.887852 & -0.583662 & -0.398514 \\
\hline 40 & 1 & -5.217930 & 0.446662 & -0.464707 \\
\hline 41 & 6 & -3.538041 & -0.883434 & -0.171001 \\
\hline 42 & 6 & 5.683599 & -1.954474 & -0.594651 \\
\hline 43 & 1 & 6.743241 & -1.835218 & -0.788812 \\
\hline 44 & 5 & 0.000188 & -0.435858 & 0.805735 \\
\hline
\end{tabular}

$\mathrm{E}_{h}=-1354.582593$ Hartree 
References

(1) Williams, A. T. R.; Winfield, S. A.; Miller, J. N. Relative Fluorescence Quantum Yields Using a Computer-Controlled Luminescence Spectrometer. Analyst 1983, 108 (1290), 1067-1071. https://doi.org/10.1039/AN9830801067.

(2) Sheldrick, G. M. A Short History of SHELX. Acta Crystallogr. Sect. A Found. Crystallogr. 2008, 64 (1), 112-122. https://doi.org/10.1107/S0108767307043930.

(3) Spek, A. L. CheckCIF Validation ALERTS: What They Mean and How to Respond. Acta Crystallogr. Sect. E 2020, 76 (1), 1-11. https://doi.org/10.1107/S2056989019016244.

(4) Stephens, P. J.; Devlin, F. J.; Chabalowski, C. F.; Frisch, M. J. Ab Initio Calculation of Vibrational Absorption and Circular Dichroism Spectra Using Density Functional Force Fields. $J$. Phys. Chem. 1994, 98 (45), 11623-11627. https://doi.org/10.1021/j100096a001.

(5) Tomasi, J.; Mennucci, B.; Cammi, R. Quantum Mechanical Continuum Solvation Models. Chem. Rev. 2005, 105 (8), 2999-3093.

(6) McLean, A. D.; Chandler, G. S. Contracted Gaussian Basis Sets for Molecular Calculations. I. Second Row Atoms, $Z=11-18$. J. Chem. Phys. 1980, 72 (10), 5639-5648. https://doi.org/10.1063/1.438980.

(7) Frisch, M. J.; Trucks, G. W.; Schlegel, H. B.; Scuseria, G. E.; Robb, Ma.; Cheeseman, J. R.; Scalmani, G.; Barone, V.; Mennucci, B.; Petersson, G. A. Gaussian 09, Revision D. 01, Gaussian. Inc. Wallingford, CT 2009.

(8) Tenderholt, A. QMForge. Stanford University: Stanford, CA, USA. 